\title{
Knowing one's future preferences: A correlated agent model with Bayesian updating
}

\section{Citation}

Bas, M. A., C. S. Signorino, and T. Whang. 2013. “Knowing One's Future Preferences: A Correlated Agent Model with Bayesian Updating." Journal of Theoretical Politics 26 (1) (May 2): 3-34. doi:10.1177/0951629813482054.

\section{Published Version}

doi:10.1177/0951629813482054

\section{Permanent link}

http://nrs.harvard.edu/urn-3:HUL.InstRepos:22547789

\section{Terms of Use}

This article was downloaded from Harvard University's DASH repository, and is made available under the terms and conditions applicable to Open Access Policy Articles, as set forth at http:// nrs.harvard.edu/urn-3:HUL.InstRepos:dash.current.terms-of-use\#OAP

\section{Share Your Story}

The Harvard community has made this article openly available.

Please share how this access benefits you. Submit a story.

\section{Accessibility}




\title{
Knowing One's Future Preferences: A Correlated Agent Model with Bayesian Updating*
}

\author{
Muhammet A. Bas ${ }^{\dagger}$ \\ Curtis S. Signorino $\ddagger$ \\ Taehee Whang ${ }^{\S}$
}

\begin{abstract}
We generalize two classes of statistical sequential incomplete information games: (1) those resembling typical signalling games, in which a single agent represents each player, allowing for information to be revealed about future play; and (2) those in which each player is represented by a set of independent agents, where moves do not reveal private information. The generalized model we develop, the Correlated Agent Model, relies on a parameter, $\rho$, which denotes the correlation between two agents' private information i.e., the extent to which a player knows the future private component of her preferences. The independent agent and single agent models are special cases, where $\rho=0$ and $\rho=1$, respectively. The model also allows $0<\rho<1$, a class of games which have not yet been considered. We apply the model to crisis bargaining and demonstrate how to estimate $\rho$, as well as parameters associated with utilities.
\end{abstract}

\footnotetext{
*We would like to thank Songying Fang, Justin Fox, Jeffrey Lewis, Kyoungwon Seo for comments and suggestions. Support from the National Science Foundation (Grant \# SES-0413381) is gratefully acknowledged.

${ }^{\dagger}$ Department of Government, Harvard University, E-mail: mbas@gov.harvard.edu

¥Department of Political Science, University of Rochester, E-mail: sign@mail.rochester.edu

$\S$ Division of International Studies, Korea University, E-mail: twng@korea.ac.kr
} 


\section{Introduction}

How do state leaders, members of Congress, voters, or consumers know what their future preferences will be? In most applications of game theory to political behavior, researchers generally assume that decision makers know their own future preferences with complete certainty. While this assumption seems reasonable in some cases (e.g., when a small number of decisions will be made over a short period of time), there may be other situations where it is implausible (e.g., when decisions will be made over a long period of time). In this paper, we examine future preferences and signaling in the context of statistical games. We develop a new approach (a Correlated Agent Model) that generalizes two major classes of models with private information. We demonstrate how to conduct statistical estimation using this new approach and apply these techniques to international crisis bargaining.

Current statistical models for crisis bargaining entail either traditional game-theoretic assumptions (Lewis and Schultz 2003; Wand 2006; Whang 2010b; Whang, McLean and Kubserski 2013) or "independent agent" assumptions(Signorino 2003; Leblang 2003; Signorino and Tarar 2006; Gent 2007; Bas, Signorino and Walker 2008; McLean and Whang 2010; Carter 2010; Bas 2012). These approaches differ in what they assume about players' uncertainty concerning their own preferences. Traditional game-theoretic models assume that each player completely knows at every point in the game how much she will value the outcomes in the model, no matter how far in the future those outcomes are. In contrast, many recent stochastic games (e.g., McKelvey \& Palfrey's Quantal Response Equilibrium (McKelvey and Palfrey 1995, 1998) and Signorino's Nash-based strategic probit models (Signorino 1999, 2003)) take an independent agent approach. Here, each player is represented by a different agent for each information set. A player's agents share the same average utility for outcomes, but have different private components that are unobserved by her fellow agents. This assumption makes intuitive sense when moves are temporally distant - e.g., I 
may not know exactly how, at some point in the future, I will value a given outcome. This assumption is also realistic when we have a reason to expect changes in a player's agents i.e., when we have reason to believe that a current agent and a successor agent will differently value a given outcome. Finally, it is reasonable to have the independent agent assumption when there are unexpected events or exogenous shocks in the course of the game that alter the utility evaluation of players.

Crucially, these two models lead to distinct implications regarding the ability of players to signal their resolve and learn from the actions of opponent players. The traditional Bayesian models allow each player to update his/her initial beliefs in the game. Since players know their own private information before the game begins, the informed player is capable of signaling his/her true 'type' and the other players fully adjust their prior assessment of their opponent's type and actions accordingly. The independent agent assumption, on the other hand, implies that players do not learn from each other's moves, since private information for each agent is unknown to, and independent of, that player's other agents farther down the tree.

In many important contexts - e.g., international conflicts involving territorial disputes, economic sanctions, or military interventions - the extent to which actors have correct understanding of their own payoffs down the game tree remains an empirical question. Rather than pitting these approaches against each other as the only two options, and as mutually exclusive options, we develop a more general approach — the Correlated Agent Model — that contains each as a special case and allows us to estimate the extent to which actors know their future preferences.

The remainder of this paper proceeds as follows. In the next section, we set up a theoretical model that generalizes both the traditional Bayesian model and the independent agent model. Following that, we derive equilibrium probabilities and develop a maximum 
likelihood estimator based on this more general model. We then present an application of our statistical model to international crisis bargaining, using data from Lewis and Schultz (2005).

\section{Future Preferences and Correlated Agents}

Game-theoretic models of signaling have been used extensively in the field of international relations. Whether the topic is crisis bargaining (Morrow 1989; Ramsay 2004), deterrence (Powell 1990; Fearon 2002), crisis escalation and inter-state conflict (Fearon 1997; Slantchev 2005), the role of domestic politics in foreign policy choices (Fearon 1994a; Mo 1995; Schultz 1998), terrorism (Overgaard 1994; Arce and Sandler 2010), or economic sanctions (Martin 1993; Drezner 1998), scholars have employed signaling models to develop theories regarding the role uncertainty plays in strategic interactions, and what tools and mechanisms are available for actors to reduce this uncertainty or to exploit it.

A very simple but functional version of a game involving signaling and belief updating is presented in Figure 1. Due to the simplicity of this model and its ability to fully capture the essence of signaling and belief updating, discrete-choice models with the same or very similar game structures have been used extensively in the literature (Fearon 1994b; Smith 1999; Schwebach 2000; Schultz 2001; Fearon 2002; Lewis and Schultz 2003; Lacy and Niou 2004; Lewis and Schultz 2005; Kurizaki 2007; Esarey, Mukherjee and Moore 2008; Fey and Ramsay 2010). Due to these desirable properties, we will also use this model to develop the statistical estimator in the next section.

[Figure 1 about here.]

The game in Figure 1 (a) represents a simple interaction between two players, $A$ and $B$. There are three decision nodes and four possible outcomes. Each player's utility for a given 
outcome has two components: an observable component that is visible to both players, and a private information component that is only revealed to the player herself. Accordingly, Player A's utility for outcome $k$ is written as $U_{k}+v_{k}$, where $U_{k}$ represents the observable component and $v_{k}$ the private component. Similarly, Player B's utility for outcome $k$ is written as $V_{k}+\nu_{k}$. We assume that $v_{k}$ and $\nu_{k}$ follow probability distributions $f(v)$ and $g(\nu)$ respectively.

The game proceeds as follows: Nature moves first, and draws $v_{k}$ and $\nu_{k}$ from their corresponding distributions and selectively reveals them to A and B. Then Player A moves, choosing between actions $a_{1}$ and $a_{2}$. If $a_{1}$ is chosen, the game ends with Outcome $1 .{ }^{1}$ If $a_{2}$ is chosen, then Player $\mathrm{B}$ chooses between $a_{3}$ and $a_{4}$. If $B$ chooses $a_{3}$, the game ends with Outcome 3. If $a_{4}$ is chosen, then Player A moves again, choosing between $a_{5}$ and $a_{6} . a_{5}$ ends the game with Outcome 5 , and $a_{6}$ results in Outcome 6.

This is a traditional two-player Bayesian game with two-sided incomplete information. We call this game the Single Agent Model (SAM) as A moves twice in the game and possesses perfect information about her utilities in both stages. Based on an appropriately specified set of utilities $\{\mathbf{U}, \mathbf{V}\}$ and the probability distributions $f(v)$ and $g(\nu)$, we can solve this game for Perfect Bayesian Equilibria (PBE). For the purpose of developing the statistical estimator later in the paper, we are especially interested in players' probabilities of choosing each action $\left\{p_{1}, p_{2}, \ldots, p_{6}\right\}$, and the probability distribution of outcomes that these probabilities imply.

We will now consider the two-agent version of the same game, shown in Figure 1 (b). This is much like the game in Figure 1 (a), except now Player A is represented by two agents, $A_{1}$ and $A_{2}$, who share the same observable utility $U_{k}$ for an outcome $\mathrm{k}$, while at the same time each agent's private component is not directly observable to the other agent. ${ }^{2}$ We will denote the private components of $A_{1}$ by $v_{k}$ as above, and $A_{2}$ 's private components by $\pi_{k}$. Like the private information components of $A_{1}$ and $B$ 's outcome utilities, $\pi_{k}$ is assumed 
to follow a continuous probability distribution, which we assume to be a standard Normal distribution.

One can think of $A_{1}$ and $A_{2}$ as literally two separate agents for Player A - e.g., different decision-making incarnations of A that emerge in the course of the game. Alternatively, one could think of this as Player A making decisions over a somewhat more extended period of time - where she has a sense at the first node what her utility is likely to be at the last node, but realizes that it may change slightly by that point.

In the typical agent specification, which we will refer to as the Independent Agent Model (IAM), the private components $v_{k}$ and $\pi_{k}$ are assumed to be independent and identically distributed. In other words, $A_{1}$ and $B$ have the same amount of uncertainty about $A_{2}$ 's choice at the end of the game. In this formulation, it is helpful to think of the game as consisting of three different players, two of which $\left(A_{1}\right.$ and $\left.A_{2}\right)$ share the same observable utilities for outcomes. Therefore, once $A_{1}$ moves, B learns nothing about A's private information and what $A_{2}$ is likely to do at the last node. In this game, there is no belief updating by any player. For $A_{1}$, knowing her own outcome payoffs does not give any information about $A_{2}$ 's payoffs because of the independence of private components. There is no belief updating on the part of $\mathrm{B}$ either, because $A_{1}$ 's choice in the game does not give any information about $A_{2}$ 's likely choice.

IAM and SAM are limit cases of a more general model. Suppose we maintain the construct of agents $A_{1}$ and $A_{2}$, but now assume that $\operatorname{cor}\left(v_{k}, \pi_{k}\right)=\rho \neq 0$ for outcomes 5 and $6 .{ }^{3}$ In contrast to IAM, in this case, B may learn something about $A_{2}$ 's private information and what $A_{2}$ is likely to do by observing $A_{1}$ 's choice, because of the non-zero correlation between the private information components of $A_{1}$ and $A_{2}$ 's outcome payoffs. We refer to this more general model as the Correlated Agent Model (CAM). IAM and SAM, with A moving twice in the game, can now be seen as special cases of CAM. IAM corresponds to $\rho=0$, while 
SAM represents the case $\rho=1$. To find the equilibrium of this model, we need to take into account the prior and posterior information $A_{1}$ and $\mathrm{B}$ have about the distribution of $\pi_{k}$. The following section derives the PBE for this model by calculating the prior and posterior probabilities that $A_{1}$ and $B$ assign to $A_{2}$ 's choice in the game.

\subsection{Correlated Agent Model}

Based on the setup of the model, we solve for the PBE of the game, which requires that players' strategies are sequentially rational given their beliefs, and their beliefs are derived from the equilibrium strategies using the Bayes' Rule whenever possible.

We first start with $A_{2}$ 's decision. In equilibrium, $A_{2}$ chooses $a_{6}$ if and only if it gives her a higher expected utility than $a_{5}$. In other words,

$$
U_{6}+\pi_{6} \geq U_{5}+\pi_{5}
$$

If we go up one decision node, $B$ chooses $a_{4}$ in equilibrium if and only if it gives her a better expected payoff. Since $B$ has uncertainty about what $A_{2}$ will do if $B$ chooses $a_{4}$, he needs to calculate $A_{2}$ 's probability of choosing $a_{6}\left(p_{6}\right)$ and $a_{5}\left(p_{5}\right)$ and weight his outcome utilities $V_{6}+\nu_{6}$ and $V_{5}+\nu_{5}$ accordingly. In other words, $B$ chooses $a_{4}$ whenever

$$
\left(1-p_{6}^{B}\right)\left(V_{5}+\nu_{5}\right)+p_{6}^{B}\left(V_{6}+\nu_{6}\right) \geq V_{3}+\nu_{3}
$$

where $p_{6}^{B}$ represents $B$ 's estimate of $A_{2}$ 's action probability after observing $a_{2}$ by $A_{1}$. Finally, in equilibrium, $A_{1}$ compares her expected payoffs from $a_{1}$ and $a_{2}$ (right and left-hand sides of inequality 3 below). Because of her uncertainty about $B$ 's and $A_{2}$ 's outcome payoffs, $A_{1}$ needs to estimate their action probabilities and use these estimated probabilities in her 
utility comparison. More formally, $A_{1}$ chooses $a_{2}$ if and only if

$$
\left(1-p_{4}\right)\left(U_{3}+v_{3}\right)+p_{4}\left(1-p_{6}^{A_{1}}\right)\left(U_{5}+v_{5}\right)+p_{4} p_{6}^{A_{1}}\left(U_{6}+v_{6}\right) \geq U_{1}+v_{1}
$$

where $p_{4}$ represents $A_{1}$ 's prediction of $B$ 's probability of choosing $a_{4}$. Similarly, $p_{6}^{A_{1}}$ represents $A_{1}$ 's estimate of $A_{2}$ 's probability of choosing $a_{6}$. Equilibrium conditions 1-3 satisfy the sequential rationality requirement of PBE given players' beliefs. We now need to make sure the beliefs are consistent with the strategies using the Bayes' Rule.

Note that in this game there are three different estimates for $A_{2}$ 's probability of choosing $a_{6}$. The first, denoted by $p_{6}$, is based on $A_{1}$ and $B$ 's prior beliefs about the probability distribution of $A_{2}$ 's private information components $\pi_{i}$ before any player makes a move. By the assumptions of the game, this prior distribution is $\operatorname{Normal}(0,1)$. After Nature reveals $A_{1}$ 's private information components $v_{i}$ to $A_{1}$, knowing $v_{i}$ gives $A_{1}$ information about $\pi_{i}$, due to the correlation $\rho$ between the two. $A_{1}$ 's updated estimate for $p_{6}$ is denoted by $p_{6}^{A_{1}}$.

Since $\pi_{i}$ and $\nu_{i}$ are not correlated, knowing his own private information components does not give $B$ any more information about $A_{2}$ choice than that is already summarized by the prior distribution of $\pi_{i}$. However, $B$ updates his prior belief after observing $A_{1}$ 's action, as $A_{1}$ 's action implicitly conveys information about the more and less likely values for $A_{2}$ 's private payoffs. This posterior probability estimate $B$ calculates after observing $A_{1}$ 's choice is denoted by $p_{6}^{B}$.

As derivations in the Appendix show, $p_{6}^{A_{1}}$ and $p_{6}^{B}$ will usually take different values. This is because $A_{1}$ possesses more information than $B$ about $A_{2}$ 's choice due to the correlation between $A_{1}$ and $A_{2}$ 's private information components. After Nature reveals $A_{1}$ 's private information components $v_{i}$ to $A_{1}, A_{1}$ uses this information to make more accurate inferences about $A_{2}$ 's action probability. $B$, on the other hand, can only update his prior estimate of 
$A_{2}$ 's choice probability indirectly by observing $A_{1}$ 's action.

For ease of presentation, we define here the following three latent variables that capture $A_{1}$ 's, $B$ 's, and $A_{2}$ 's choices:

$$
\begin{aligned}
Y_{A_{1}} & =\left(1-p_{4}\right)\left(U_{3}+v_{3}\right)+p_{4}\left(1-p_{6}^{A_{1}}\right)\left(U_{5}+v_{5}\right)+p_{4} p_{6}^{A_{1}}\left(U_{6}+v_{6}\right)-U_{1}-v_{1} \\
Y_{B} & =\left(1-p_{6}^{B}\right)\left(V_{5}+\nu_{5}\right)+p_{6}^{B}\left(V_{6}+\nu_{6}\right)-V_{3}-\nu_{3} \\
Y_{A_{2}} & =U_{6}-U_{5}+\pi_{6}-\pi_{5}
\end{aligned}
$$

Note that in equilibrium, $A_{1}$ chooses $a_{2}$ if and only if $Y_{A_{1}} \geq 0$. Similarly, $B$ chooses $a_{4}$ if and only if $Y_{B} \geq 0$, and $A_{2}$ chooses $a_{6}$ if and only if $Y_{A_{2}} \geq 0$. Defined this way, $Y_{i}$ are random variables as they are functions of Normally distributed private information components of each player. Proposition 1 below defines the relevant probabilities that characterize the PBE.

Proposition 1 The equilibrium of CAM is characterized by the following probabilities:

$$
\begin{aligned}
p_{6} & =\operatorname{Pr}\left(Y_{A_{2}} \geq 0\right) \\
p_{6}^{A_{1}} & =\operatorname{Pr}\left(Y_{A_{2}} \geq 0 \mid v_{5}, v_{6}\right) \\
p_{6}^{B} & =\operatorname{Pr}\left(Y_{A_{2}} \geq 0 \mid Y_{A_{1}} \geq 0\right) \\
p_{4} & =\operatorname{Pr}\left(Y_{B} \geq 0 \mid Y_{A_{1}} \geq 0\right) \\
p_{2} & =\operatorname{Pr}\left(Y_{A_{1}} \geq 0 \mid Y_{A_{1}} \geq 0 ; Y_{B} \geq 0\right)
\end{aligned}
$$

where $p_{6}$ represents $A_{1}$ 's and $B$ 's prior estimates, and $p_{6}^{A_{1}}$ and $p_{6}^{B}$ their posterior estimates respectively, of $A_{2}$ 's probability of choosing $a_{6} \cdot p_{2}$ and $p_{4}$ represent $A_{1}$ 's probability of choosing 
$a_{2}$, and $B$ 's probability of choosing $a_{4}$, respectively. The exact probabilities are as follows:

$$
\begin{aligned}
p_{6}= & \Phi\left(\frac{U_{6}-U_{5}}{\sqrt{2}}\right) \\
p_{6}^{A_{1}}= & \Phi\left(\frac{U_{6}-U_{5}+\rho \epsilon}{\sqrt{2-2 \rho^{2}}}\right) \\
p_{6}^{B}= & \left.\left.\frac{E_{\epsilon}\left[\Phi\left(\frac{U_{6}-U_{5}+\rho \epsilon}{\sqrt{2-2 \rho^{2}}}\right) \Phi\left(\frac{\left(1-p_{4}\right) U_{3}+p_{4}\left[\left(1-\bar{p}_{6}^{A_{1}}\right) U_{5}+\bar{p}_{6}^{A_{1}} U_{6}\right]-U_{1}+\frac{p_{4}\left[2 \bar{p}_{6}^{A_{1}}-1\right] \epsilon}{2}}{\sqrt{\left(1-p_{4}\right)^{2}+\frac{p_{4}^{2}}{2}+1}}\right)\right]}{p_{2}}\right)\right] \\
p_{4}= & \Phi\left(\frac{\left(1-p_{6}^{B}\right) V_{5}+p_{6}^{B} V_{6}-V_{3}}{\sqrt{\left(1-p_{6}^{B}\right)^{2}+\left(p_{6}^{B}\right)^{2}+1}}\right) \\
p_{2}= & E_{\epsilon}\left[\Phi\left(\frac{\left(1-p_{4}\right) U_{3}+p_{4}\left[\left(1-\bar{p}_{6}^{A_{1}}\right) U_{5}+\bar{p}_{6}^{A_{1}} U_{6}\right]-U_{1}+\frac{p_{4}\left[2 \bar{p}_{6}^{A_{1}}-1\right] \epsilon}{2}}{\sqrt{\left(1-p_{4}\right)^{2}+\frac{p_{4}^{2}}{2}+1}}\right)\right]
\end{aligned}
$$

where $\epsilon=v_{6}-v_{5}$ is a (normally distributed) random variable and $E_{\epsilon}$ is the expectation operator for $\epsilon$.

Proof of Proposition 1 is in the Appendix. The following two claims establish that IAM and SAM are special cases of CAM when $\rho=0$ and $\rho=1$, respectively, and are proved in the Appendix.

Claim 1 When $\rho=0$, the Correlated Agent Model reduces to the Independent Agent Model 
with the following action probabilities:

$$
\begin{aligned}
p_{6}=p_{6}^{B}=p_{6}^{A_{1}} & =\Phi\left(\frac{U_{6}-U_{5}}{\sqrt{2}}\right) \\
p_{4} & =\Phi\left(\frac{p_{6}^{B} V_{6}+\left(1-p_{6}^{B}\right) V_{5}-V_{3}}{\sqrt{1+\left(p_{6}^{B}\right)^{2}+\left(1-p_{6}^{B}\right)^{2}}}\right) \\
p_{2} & =\Phi\left(\frac{\left(1-p_{4}\right) U_{3}+p_{4}\left[\left(1-p_{6}^{A_{1}}\right) U_{5}+p_{6}^{A_{1}} U_{6}\right]-U_{1}}{\sqrt{\left(1-p_{4}\right)^{2}+p_{4}^{2}\left(1-p_{6}^{A_{1}}\right)^{2}+p_{4}^{2}\left(p_{6}^{A_{1}}\right)^{2}+1}}\right)
\end{aligned}
$$

Claim 2 When $\rho=1$, the Correlated Agent Model reduces to the Single Agent Model with the following action probabilities:

$$
\begin{aligned}
p_{4} & =\Phi\left(\frac{p_{6}^{B} V_{6}+\left(1-p_{6}^{B}\right) V_{5}-V_{3}}{\sqrt{1+\left(p_{6}^{B}\right)^{2}+\left(1-p_{6}^{B}\right)^{2}}}\right) \\
p_{2} & \left.=1-\Phi_{2}\left[\frac{U_{1}-\left(1-p_{4}\right) U_{3}-p_{4} U_{5}}{\sqrt{p_{4}^{2}+1+\left(1-p_{4}\right)^{2}}}, \frac{U_{1}-\left(1-p_{4}\right) U_{3}-p_{4} U_{6}}{\sqrt{p_{4}^{2}+1+\left(1-p_{4}\right)^{2}}}, \frac{1+\left(1-p_{4}\right)^{2}}{p_{4}^{2}+1+\left(1-p_{4}\right)^{2}}\right]\right) \\
p_{6}^{B} & =\Phi_{2}\left[\frac{U_{6}-U_{5}}{\sqrt{2}}, \frac{p_{4} U_{6}-U_{1}+\left(1-p_{4}\right) U_{3}}{\sqrt{p_{4}^{2}+1+\left(1-p_{4}\right)^{2}}}, \frac{p_{4}}{\sqrt{2\left(1+\left(1-p_{4}\right)^{2}+p_{4}^{2}\right)}}\right] / p_{2}
\end{aligned}
$$

Before using CAM for empirical analysis, we need to know that it satisfies certain statistical properties. In the Appendix, we conduct two sets of Monte Carlo (MC) experiments to establish the unbiasedness and consistency of CAM. For this purpose, we first generated simulated samples for a variety of outcome utility and correlation values, and for each configuration, we approximated the sampling distribution of CAM estimation parameters. Our results suggest that CAM provides unbiased estimates, and the standard errors of the estimates approach zero as the sample size increases, indicating consistency. The second 
MC experiment provides a comparison of CAM, SAM, and IAM in a variety of situations ranging from very low to very high correlation values, and aims to show that, overall, CAM performs better than SAM and IAM. We find that CAM is a superior approach to both when the underlying correlation between the private information components of Player A's two agents is not known. Second, CAM also does at least as well as IAM when this correlation is very close to zero, and as well as SAM when the correlation is close to one. For moderate correlation values, CAM clearly outperforms both.

We now turn to an empirical application of the CAM estimator we just developed.

\section{$3 \quad$ Empirical Application}

In this section, as an empirical application of CAM, we analyze factors that affect interstate crisis initiation and escalation, using data from Lewis and Schultz (2005). Lewis and Schultz (2005) analyze the factors that affect a state leader's decision to initiate an international crisis and escalate it militarily. In their analysis, they employ a fully structural statistical model using a traditional Bayesian updating game that assumes $\rho=1$, resembling SAM in ways discussed above. Figure 2 displays the extensive form of the crisis bargaining game that forms the basis of the coding of the dependent variable - outcome of the crisis interaction - in their analysis. State A first decides either to challenge the status quo with an explicit

threat of using force in the case of the opponent state's resistance, or to stay with the status quo (Status Quo: SQ). Upon A's challenge, State B decides either to make a concession (Acquiescence: ACQ) or to resist the demand of A. If B resists, A has a final choice of either backing down from the initial challenge (Back Down: BD) or fighting (Stand Firm: SF). There are four mutually exclusive outcomes: SQ, ACQ, BD, and SF, and corresponding utilities of $\mathrm{A}$ and B: $S Q_{A}, A C Q_{A}, B D_{A}$, and $S F_{A}$ for $\mathrm{A}$ and $S Q_{B}, A C Q_{B}, B D_{B}$, and $S F_{B}$ 
for B. [Figure 2 about here.]

[Table 1 about here.]

The data consist of 93 dyadic crisis cases from the period 1919 to 1931, comprising both Militarized Interstate Dispute data (MID) and International Conflict Behavior data (ICB). The number of observations is 2187 with the addition of SQ cases. The distribution of crisis outcomes is $\mathrm{SQ}=2094, \mathrm{ACQ}=44, \mathrm{BD}=12$, and $\mathrm{SF}=37$ observations. Table 1 shows the variables and measures used in our empirical analysis, and their sources. To briefly summarize the model specification in general terms, State A's SQ utility is associated with the maximum number of years since State 1 or 2 joined the international system after WWI (measured by MaxAge). ACQ utilities of A and B are modeled by three variables: the similarity of the two states' strategic interests (Alliance), domestic stability in B (CivilWar ${ }_{B}$ ), and whether the two states share a common land border or are separated by less than 150 miles of water (Contiguous). Following the democratic peace literature, A's democracy level $\left(\right.$ Democracy $\left._{A}\right)$ is included in the BD utility of A. Three covariates are included in A's and B's SF utilities. $S F_{A}$ is related to the democracy score of A $\left(\right.$ Democracy $\left._{A}\right)$, the relative capability ratio of A to B ( CapShare $\left._{A}\right)$, and the economic development level of A (Develop $A$ ), while $S F_{B}$ is associated with capability ratio, democracy, and economic development of B ( CapShare $_{A}$, Democracy $_{B}$, and Develop $p_{B}$ ). All the variables are included in their corresponding utilities using a linear functional form. For instance, the observable component of A's SQ utility can be expressed as $U(A, S Q)=\beta_{0}+\beta_{1}$ MaxAge, where $\hat{\beta}_{0}$ and $\hat{\beta}_{1}$ are estimated by the model.

Identification is an important concern here because the amount of information in the data (i.e., four mutually exclusive outcomes) is less than the number of parameters to estimate (i.e., theoretically eight utilities). An obvious way to address this problem is to assume that each utility is a function of regressors and thereby to increase variation in the data. While we enhance the model's ability to distinguish outcomes with explanatory variables as displayed 
in Table 1, we also follow suggestions made in the Appendix of Lewis and Schultz (2003). In particular, we use normalization of the utilities by addition and multiplication while keeping them intact with optimal choices drawn from the equilibrium probabilities. We make an assumption regarding State A's utilities such that the constant terms of $S Q_{A}$ and $B D_{B}$ are equal to zero. Moreover, no regressor is included in all utilities of any state. Finally, $S Q_{B}$ is not estimated because it is not considered in equilibrium calculation. There is no loss of generality as a result of these restrictions. ${ }^{4}$

CAM aims to improve SAM (e.g., Lewis and Schultz 2003) and IAM (e.g., Signorino 1999, 2003) by parameterizing $\rho$. In the data analysis, $\rho$ is defined as the correlation between the private information components of State A's two agents in the first and third decision nodes. In a SAM model, as in Lewis and Schultz (2003, 2005), State A knows her SF and BD payoffs perfectly at any point in the game. In other words, in our model's terms, $\rho$ is assumed to be one. The primary purpose of our estimation of CAM here is to find out if this assumption of SAM is too restrictive in analyzing international crisis bargaining. In order to facilitate a comparison between SAM and CAM, we borrow Lewis and Schultz's main specification for the utilities and add $\rho$ as an additional parameter to be estimated. As noted previously, as $\rho$ approaches zero the data-generating process approximates IAM where no meaningful belief updating occurs, while as $\rho$ approaches one, the data generating-process behind international crisis bargaining follows SAM, which permits signaling and belief updating.

We model $\rho$ as a link function of regressors such that

$$
\rho=\frac{\exp (X \gamma)-1}{\exp (X \gamma)+1}
$$

where $\gamma$ is a vector of coefficients of the regressors $X$. This way, $\rho$ is well bounded between -1 and 1. We select two regressors, the log of A's total population (Population $A$ ) and A's level 
of democracy (Democracy $A$ ). When we suppose, for instance, that the correlation between the private information components of A's agents is systematically associated with the level of A's total population, $\rho$ becomes

$$
\rho=\frac{\exp \left(\gamma_{0}+\gamma_{1} \text { Population }_{A}\right)-1}{\exp \left(\gamma_{0}+\gamma_{1} \text { Population }_{A}\right)+1}
$$

and we estimate $\hat{\gamma}_{0}$ and $\hat{\gamma}_{1}$. Then, the coefficient of Population $_{A}$ helps us infer how much State A is certain about her own preference over crisis bargaining outcomes down the game tree. We choose the population and democracy of the challenger state A to understand $\rho$ because they are good proxies to characterize traditional military power and international crisis management in the interwar period. ${ }^{5}$ It is reasonable to hypothesize that, if State A has a large population, the degree of uncertainty between $\mathrm{A}_{1}$ and $\mathrm{A}_{2}$ is likely to decline and, hence, the private terms of two agents should be more correlated, compared with the cases with small population challengers ${ }^{6}$. This reasoning leads us to expect a positive coefficient for the population variable. On the other hand, Democracy ${ }_{A}$ is expected to have a negative effect on $\rho$ because elected officials responsible for foreign policies are unlikely to share identical preferences in a democratic country where leadership changes on a regular basis. Democracy $_{A}$ and Democracy are also included in each state's Stand Firm utilities to control for the effect of regime type on states' crisis preferences. We also include another indicator of states' power in their utilities, which is the state's share of military capabilities in the particular dyad $\left(\right.$ CapShare $\left._{A}\right){ }^{7}$

[Table 2 about here.]

[Table 3 about here.]

The estimation findings are presented in Tables $2-3 .^{8}$ The first, second and third columns denote the outcomes, utilities, and regressors assigned to the utilities, followed by 
the estimation results, coefficients and standard errors of SAM (Lewis and Schultz 2005). ${ }^{9}$ CAM1 results in the sixth and seventh columns use the same data and utility specifications of SAM except that the estimation model is now the correlated agent model where $\rho$ is a

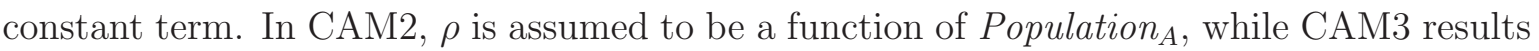
use Population $_{A}$ and Democracy ${ }_{A}{ }^{10}$

The findings of CAM1, CAM2 and CAM3 confirm that most of the coefficients have the same signs as those from SAM. First, the positive and significant coefficient of MaxAge implies that younger states mostly created in the wake of WWI are more likely to be revisionist challengers. Second, the negative and significant Democracy $A$ and constant term (except for CAM2) of $B D_{A}$ implies that audience costs exist in that $\mathrm{A}$ is significantly worse off after backing down than after maintaining the status quo. Audience costs also increase, or the probability of $B D_{A}$ decreases, if $\mathrm{A}$ is democratic. Third, the positive CapShare $_{A}$ and negative Democracy ${ }_{A}$ coefficients imply that A's utility $S F_{A}$ in the SF outcome increases as A's military advantage over B increases, and as A is less democratic. All three CAM results show that Develop $_{A}$ is statistically insignificant in contrast to SAM results. All three variables and the constant term in $S F_{B}$ fail to pass conventional criteria for statistical significance. Fourth, while SAM has no significant coefficients in ACQ, whether A or B, CAM1 finds some of them significant at a $10 \%$ significance level. The negative coefficient of Alliance and the positive coefficient for CivilWar ${ }_{B}$ imply that A's ACQ payoff, $A C Q_{A}$, increases as A and B have less similarity in their alliance portfolios, and as B is involved in a civil war. Moreover, the positive coefficient of Alliance implies that B's ACQ payoff, $A C Q_{B}$, increases as A and B have similar alliance portfolios. However, these variables lose significance in CAM2 and only Alliance in $A C Q_{A}$ survives in CAM3.

In order to compare the two models, we first conduct a Likelihood Ratio (LR) test between SAM and CAM models to examine how much CAM models improve on the fit of 
the SAM model. LR test is an appropriate choice here as SAM is a special case of CAM with a restriction of $\rho=1$. The test aims to find out is relaxing this restriction results in a significant improvement in model fit. The statistic follows a $\chi^{2}$ distribution with degrees of freedom equal to the extra parameters used in modeling $\rho$. The LR statistic for the test between SAM and CAM1 is $-2 *(-418.9+416.64)=4.52$ and the corresponding p-value is 0.0335. Thus, the results suggest that modeling $\rho$ significantly increases the model fit and our understanding of crisis bargaining. In addition, we compared the two approaches based on their predictive performance. We find that CAM clearly outperforms SAM in terms of the percentage of Resist and Fight decisions correctly predicted (SAM: 59\% for Fight, 56\% for Resist; CAM 76-79\% for Fight, 56-62\% for Resist). For the initial Challenge decision, both models correctly predict approximately the same percentage of cases (96\%).

Turning to the estimation of $\rho$, CAM1 estimates $\rho$ as a constant term. While the estimated $\rho$ fails to be significant, this is not surprising. This specification is only meant to serve as a baseline, as estimating $\rho$ as a constant term supposes that all challenger states in the data have on average the same level of information about their future preferences, which is unrealistic. To capture the potential variations across challenger states in the level of correlation between the two agents' private information, CAM2 and CAM3 incorporate Population $_{A}$ and Democracy $A$ into the $\rho$ specification. CAM2 provides a positive and significant coefficient of A's level of total population, Population $_{A}(\mathrm{p}$-value $=0.0540)$. This positive association implies that the correlation between private information of $A_{1}$ and $A_{2}$ increases as A's total population increases. When we plug in the average value of $\operatorname{Population}_{A}$ while all other variables are held constant at their mean values, the predicted value of $\rho$ is only 0.26. However, the $\rho$ prediction increases up to 0.99 at the maximum Population $_{A}$ value. In the interwar period, there is no doubt that population was one of the most important components of what is often called "hard power." It stands to reason that the sheer size of a 
country's manpower should be directly related to its economic output and military resources. Therefore, a challenger state with a larger population should have better knowledge of what she will prefer far down the game tree than a challenger with a smaller population. When Democracy $_{A}$ is added to the CAM2 specification, CAM3 also produces similar results. In particular, Population $_{A}$ remains robust with a positive direction. The positive coefficient of Democracy $_{A}$ implies that as State A is democratic, the correlation of stochastic components of SF utilities between its agents increases. While this does not seem to be consistent with our expectation, it fails to reach statistical significance at conventional levels.

[Figure 3 about here.]

Figure 3 displays the effect of $\rho$ on states' predicted action probabilities, the predicted probability of outcomes, and the amount of belief updating in international crises. We plot the probability that State B resists upon receiving a challenge from State A (Pr(Resist): solid line) and the probability that a final outcome of the crisis is ACQ (Pr(ACQ): dotted line) as a function of Population $_{A}$. Remaining variables are set to their mean values. We select the estimates of CAM2 instead of those of CAM3 as Democracy $A$ also appears in SF and BD outcome utilities. Because the democracy variable is included both in $\rho$ and other utilities in CAM3, it is difficult to separate out the effect of $\rho$ on the choice and action probabilities. The x-axis of Figure 3 display values within two standard deviations away from the mean of Population . $^{2}$

Figure 3 shows that as Population $_{A}$ increases, $\operatorname{Pr}$ (Resist) decreases and, hence, $\operatorname{Pr}(\mathrm{ACQ})$ increases. As the log of State A's total population increases from 5.41 (minimum) to 13.15 (maximum), the probability that State B opts for resistance upon a challenge decreases on average by $11 \%$, which in turn results in a decrease of $9 \%$ in the probability that the ACQ outcome is reached. From Table 3, recall that the population of State A is positively associated with $\rho$. Thus, we can interpret that $\rho$ is also negatively associated with $\operatorname{Pr}($ Resist) 
and positively associated with $\operatorname{Pr}(\mathrm{ACQ})$.

The role of $\rho$ is critical in understanding the findings in Figure 3 since the effects of Population $_{A}$ on state behavior works through $\rho$. This correlation parameter explains the extent to which an informational mechanism is successfully established between State A and B. We know that as the value of $\rho$ increases, the crisis bargaining game gets closer to a traditional Bayesian game, which involves knowledge being common to both State A and B. As the agents of State A share their preferences and the crisis bargaining game reduces to SAM, we expect that State B should take the act of challenge by the first agent of State A seriously. That is, State B should pay more attention to the message conveyed by State A's challenge. State B is more likely to update her initial assessment of State A's resolve than in the case in which the bargaining game reduces to the independent agent game.

State B's prior belief, her estimate of the challenger's probability of standing firm before $\mathrm{B}$ observes a challenge, is given by $p_{6}$ or equation (4) in Proposition 1 . Upon observing a challenge, State B adjusts her prior belief and forms a posterior belief, which is the conditional probability that State A will stand firm given that State A challenges, i.e., $\operatorname{Pr}($ Fight $\mid$ Challenge $)=p_{6}^{B}$ or equation (6) in Proposition 1. The difference between the posterior and prior, $\operatorname{Pr}($ Fight $\mid$ Challenge $)-\operatorname{Pr}($ Fight $)=p_{6}^{B}-p_{6}$, measures the amount of belief updating by B. As Figure 3 depicts, the amount of belief updating tends to increase

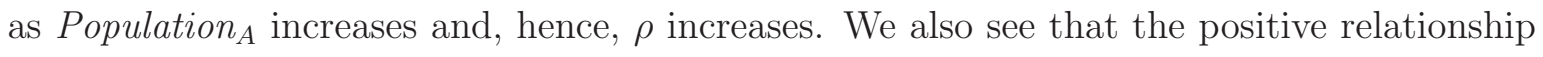
between $\rho$ and belief updating influences State B's choice at her decision-making node. State $\mathrm{B}$ is less likely to resist because State B knows that there is less uncertainty between the two agents of State A (high $\rho$ ). Compared with the opposite case of low $\rho$ in which State B has little to learn from the challenge, signaling can be effective when the value of $\rho$ is high. The act of challenge is more likely to be credible in terms of revealing the challenger's resolve. This effective informational mechanism can affect state behavior, decreasing the probability 
of Resist and increasing the probability of $A C Q$. In sum, in this particular example we considered, the larger the population of State A, the larger the probability that State A achieves concessions from B, because of more effective signalling.

\section{Conclusion}

In this paper, we developed a statistical estimator that integrates two existing models: a model that makes traditional Bayesian updating assumptions (Single Agent Model) and a model with no belief updating (Independent Agent Model). As Lewis and Schultz (2003) argued, one notable difference between the single and independent agent models is the extent to which belief updating is allowed. Because the former model assumes that players have no uncertainty over their own utilities while having uncertainty over their opponent's utilities, the informed mover has a chance to signal her type. In the independent agent model, on the other hand, signaling is impossible simply because each agent of the player has private information that is not observed by the fellow agents. Under these conditions, opponents are unable to update their prior beliefs and infer the type of the first player, because private information components are independent from each other.

While the two models stem from distinct theoretical frameworks, their differences can be summarized in terms of whether players have complete knowledge regarding their future utilities in the game. Our Correlated Agent Model estimates the extent to which players know their future preferences farther down the game tree. We do not assume, as is common in traditional Bayesian signaling models, that players know all their future utilities before the game begins. Rather, we estimate an additional parameter $\rho$ that informs us about the extent to which players know their future payoffs. Insofar as it makes no assumptions regarding the value of $\rho$, the correlated agent model absorbs single agent models (where $\rho=$ 
1) and independent agent models (where $\rho=0$ ) into a single framework. Moreover, Monte Carlo analyses demonstrate that the correlated agent model is unbiased and consistent, and outperforms the other two models in a variety of situations with different levels of correlation between actors' private information at different points in time.

Finally, using our approach, we reanalyzed a recent study by Lewis and Schultz (2005) on interstate crisis bargaining during the interwar period. While maintaining their specification for actors' utilities from the outcomes of the crisis interaction, we estimated $\rho$ as a measure of a challenger state's knowledge of her future preferences if the target state resists her challenge. In doing so, we assumed that the extent of this knowledge is a function of the state's economic development and total population. Our findings show that these factors have significant effects on how much a state knows about its future preferences: as a challenger state has a larger size of its manpower, it tends to have a clearer picture of its preferences over what to do if its target decides to resist a challenge.

\section{Notes}

${ }^{1}$ We reference each outcome by the subscript of the action that leads to it.

${ }^{2}$ The assumption that the two agents of Player A share the same observable utility is not crucial and can be relaxed without significantly changing any of the derivations. We keep this assumption to facilitate a direct comparison of the three models we consider in this paper.

${ }^{3}$ We assume that $\operatorname{cor}\left(v_{5}, \pi_{5}\right)=\operatorname{cor}\left(v_{6}, \pi_{6}\right)$. Although a model with a more general correlation structure can also be derived, we omit it here due to the additional complexity it 
introduces to the derivation of probabilities without providing new theoretical insights that would justify the additional complexity. In addition, we do not consider correlations across different outcomes for a given player. For models with within-player correlation, see Whang $(2010 a)$.

${ }^{4}$ We also de-mean the variables used in analysis to (1) make our specification consistent with that of Lewis and Schultz and (2) make it easier to interpret the results (e.g., the constant terms will have intuitive meaning because they represent the average utility of outcomes when all other variables are equal to their mean values).

${ }^{5}$ We also ran a number of different specifications for the correlation parameter using alternative indicators for state power, which are not reported here. Our substantive findings remain the same. These results are available upon request.

${ }^{6}$ If we view $\mathrm{A} 1$ and $\mathrm{A} 2$ as the representatives of the median position regarding the crisis within state A, and if we assume that individual voters' positions on the crisis issue can shift randomly over time, the likelihood of resulting large shifts in the median voter's position (hence, the preferences of A2) over time will vary depending on the population size. In particular, in larger populations, the median position will be more robust to random changes and less likely to shift over time, and hence A1's uncertainty about A2's preferences will be lower.

${ }^{7}$ In order to strengthen the identification of our model and help with convergence, we do not include the same measure of power in both the utilities and the correlation parameter. Population $_{A}$ and CapShare $A$ are highly correlated, supporting our assumption that they are proxies for the same concept. Our substantive results do not change if we include the same measure of power in both parameters, but our estimates become more fragile. These results are available upon request. 
${ }^{8}$ Two things are worth noting at this point. First, we numerically verified whether there are multiple equilibria in any of our estimation results. For all estimated utilities, we found a unique solution. Second, Lewis and Schultz $(2005,20)$ give additional preference restrictions such that "the constant associated with $A C Q_{A}$ to be greater than zero (or $S Q_{A}$ ) and the constant associated with $A C Q_{B}$ to be less than zero (or $B D_{B}$ )." While this may be a sensible assumption from a theoretical perspective, we do not assume such restrictions. It is not appropriate to suppose a priori that the counter-intuitive situations will not occur with positive probability because they should not occur theoretically. It would be more sensible to leave room for such cases in a statistical model. For detailed discussion regarding the assumption of preference ordering in fully structural statistical models, see Whang (2009).

${ }^{9}$ Although not reported here, we also estimated an IAM model. The fit of IAM in our sample was significantly worse than CAM and SAM, and therefore we chose not to include it here for space reasons. The results are available upon request.

${ }^{10}$ While space constraints prevent us from reporting these results, when we include only Democracy $_{A}$ to model $\rho$, Democracy ${ }_{A}$ is not statistically significant at conventional levels.

\section{References}

Arce, Daniel G. and Todd Sandler. 2010. "Terrorist Spectaculars: Backlash Attacks and the Focus of Intelligence." Journal of Conflict Resolution 54:354.

Bas, Muhammet A. 2012. "Democratic Inefficiency? Democracy and Suboptimal Choices in International Politics." Journal of Conflict Resolution 56(6):799-824.

Bas, Muhammet A., Curtis S. Signorino and Robert W. Walker. 2008. "Statistical Back- 
wards Induction: A Simple Method for Estimating Recursive Strategic Models." Political Analysis 16(1):21-40.

Carter, David. 2010. "The Strategy of Territorial Conflict." American Journal of Political Science 54:969-987.

Drezner, Daniel W. 1998. "Conflict Expectations and the Paradox of Economic Coercion." International Studies Quarterly 42(4):709-731.

Esarey, Justin, Bumba Mukherjee and Will H. Moore. 2008. "Strategic Interaction and Interstate Crises: A Bayesian Quantal Response Estimator for Incomplete Information Games." Political Analysis 16:250-273.

Fearon, James D. 1994a. "Domestic Political Audiences and the Escalation of International Disputes." The American Political Science Review 88:577-592.

Fearon, James D. 1994b. "Signaling versus the Balance of Power and Interests: An Empirical Test of a Crisis Bargaining Model." Journal of Conflict Resolution 38:236-269.

Fearon, James D. 1997. "Signaling Foreign Policy Interests: Tying Hands versus Sinking Costs." Journal of Conflict Resolution 41:68-90.

Fearon, James D. 2002. "Selection Effects and Deterrence." International Interactions 28:529.

Fey, Mark and Kris Ramsay. 2010. "Uncertainty and Incentives in Crisis Bargaining: GameFree Analysis of International Conflict." Forthcoming at American Journal of Political Science.

Gent, Stephen. 2007. "Strange Bedfellows: The Strategic Dynamics of Major Power Military Interventions." Journal of Politics 4:1089-1102. 
Kurizaki, Shuhei. 2007. "Efficient Secrecy: Public versus Private Threats in Crisis Diplomacy." American Political Science Review 101:543-558.

Lacy, Dean and Emerson M. S. Niou. 2004. "A Theory of Economic Sanctions and Issue Linkage: The Roles of Preferences, Information, and Threats." Journal of Politics $66(1): 25-42$.

Leblang, David. 2003. "To Defend or to Devalue: The Political Economy of Exchange Rate Policy." International Studies Quarterly pp. 533-559.

Lewis, Jeffrey and Kenneth Schultz. 2003. "Revealing preference: Empirical Estimation of a Crisis Bargaining Game with Incomplete Information." Political Analysis 11:345-367.

Lewis, Jeffrey and Kenneth Schultz. 2005. "Estimating State Preferences in International Crises: Promise and Limitations in Fully Structural Models.". Unpublished Manuscript, UCLA \& Stanford University.

Martin, Lisa L. 1993. "Credibility, Costs, and Institutions: Cooperation on Economic Sanctions." World Politics 45:406-432.

McKelvey, R. D. and T. R. Palfrey. 1995. "Quantal Response Equilibrium for Normal Form Games." Games and Economic Behavior 10:6-38.

McKelvey, R. D. and T. R. Palfrey. 1998. "Quantal Response Equilibrium for Extensive Form Games.” Experimental Economics 1:9-41.

McLean, Elena and Taehee Whang. 2010. "Friends or Foes? Major Trading Partners and the Success of Economic Sanctions." International Studies Quarterly 2:427-447.

Mo, Jongryn. 1995. "Domestic Institutions and International Bargaining: The Role of Agent Veto in Two-Level Games." American Political Science Review 89:914-24. 
Morrow, James D. 1989. "Capabilities, uncertainty, and resolve: A limited information model of crisis bargaining." American Journal of Political Science 33:941-72.

Overgaard, Per Baltzer. 1994. "The Scale of Terrorist Attacks as a Signal of Resources." Journal of Conflict Resolution 38:452-478.

Powell, Robert. 1990. Nuclear Deterrence Theory: The Search for Credibility. New York: Cambridge University Press.

Ramsay, Kristopher W. 2004. "Politics at the Waters Edge: Crisis Bargaining and Electoral Competition." Journal of Conflict Resolution 48:459-486.

Schultz, Kenneth. 1998. "Domestic Opposition and Signaling in International Crisis." American Political Science Review 94:829-44.

Schultz, Kenneth. 2001. "Looking for Audience Costs." Journal of Conflict Resolution 45:3260.

Schwebach, Valerie. 2000. Sanctions as Signals: A Line in the Sand or a Lack of Resolve? In Sanctions as Economic Statecraft: Theory and Practice, ed. Steve Chan and Cooper Drury. New York, NY: Palgrave pp. 187-211.

Signorino, Curtis. 1999. "Strategic Interaction and the Statistical Analysis of International Conflict." American Political Science Review 93:279-98.

Signorino, Curtis. 2003. "Strategy and Uncertainty in Discrete Choice Models." Political Analysis 11:316-344.

Signorino, Curtis and Ahmer Tarar. 2006. "A Unified Theory and Test of Extended Immediate Deterrence." American Journal of Political Science 50:586-605. 
Slantchev, Branislav L. 2005. "Military Coercion in Interstate Crises." American Political Science Review 99:533-47.

Smith, Alastair. 1999. "Testing Theories of Strategic Choice: The Example of Crisis Escalation." American Journal of Political Science 43:1254-1283.

Wand, Jonathan. 2006. "Comparing Models of Stragic Choice: The Role of uncertainty and signaling." Political Analysis 14:101-120.

Whang, Taehee. 2009. "Uncertainty and Learning in Statistical Strategic Models.". Unpublished Manuscript, Korea University.

Whang, Taehee. 2010a. "Empirical Implications of Signaling Models: Estimation of Belief Updating in International Crisis Bargaining." Political Analysis 18(3):381-402.

Whang, Taehee. 2010b. "Structural estimation of economic sanctions: From initiation to outcomes." Journal of Peace Research 47(5):561-573.

Whang, Taehee, Elena V. McLean and Douglas W. Kubserski. 2013. "Coercion, Information, and the Success of Sanction Threats." American Journal of Political Science 57(1):65-81. 


\section{A Appendix}

\section{A.1 Proof of Proposition 1}

First, $A_{1}$ and $B$ 's prior estimate of $A_{2}$ 's choice probability, $p_{6}$, is equal to

$$
\begin{aligned}
p_{6} & =P\left(U_{6}+\pi_{6} \geq U_{5}+\pi_{5}\right) \\
& =P\left(\pi_{5}-\pi_{6} \leq U_{6}-U_{5}\right) \\
& =\Phi\left(\frac{U_{6}-U_{5}}{\sqrt{2}}\right)
\end{aligned}
$$

because $\pi_{i} \sim N(0,1)$.

To calculate $p_{4}=\operatorname{Pr}\left(Y_{B} \geq 0\right)$, observe that $Y_{B}$ is a linear function of three independent normally distributed variables $\nu_{3}, \nu_{5}$, and $\nu_{6}$, plus a constant. Therefore, $Y_{B}$ is also normally distributed, with mean $\left(1-p_{6}^{B}\right) V_{5}+p_{6}^{B} V_{6}-V_{3}$ and variance $\left(1-p_{6}^{B}\right)^{2}+\left(p_{6}^{B}\right)^{2}+1$. Hence,

$$
p_{4}=\Phi\left(\frac{\left(1-p_{6}^{B}\right) V_{5}+p_{6}^{B} V_{6}-V_{3}}{\sqrt{\left(1-p_{6}^{B}\right)^{2}+\left(p_{6}^{B}\right)^{2}+1}}\right)
$$

The following proposition will be useful in deriving $p_{6}^{A_{1}}$ and $p_{6}^{B}:{ }^{11}$

Proposition 2 Let $Z$ be a multivariate normal distribution and partition $Z$ such that mean and variance are defined as follows.

$$
Z=\left(\begin{array}{c}
Z_{1} \\
Z_{2}
\end{array}\right) \sim N\left(\left(\begin{array}{l}
\mu_{1} \\
\mu_{2}
\end{array}\right),\left(\begin{array}{ll}
V_{11} & V_{12} \\
V_{21} & V_{22}
\end{array}\right)\right)
$$


Then, the conditional distribution of $Z_{1}$ given $Z_{2}$ is $N\left(\mu_{1 \mid 2}, V_{1 \mid 2}\right)$ where

$$
\begin{aligned}
& \mu_{1 \mid 2}=\mu_{1}+V_{12} V_{22}^{-1}\left(Z_{2}-\mu_{2}\right) \\
& V_{1 \mid 2}=V_{11}-V_{12} V_{22}^{-1} V_{21} .
\end{aligned}
$$

One way to calculate $p_{6}^{A_{1}}=\operatorname{Pr}\left(Y_{A_{2}} \geq 0 \mid v_{5}, v_{6}\right)$ is by deriving the conditional distribution $\operatorname{Pr}\left(Y_{A_{2}} \mid v_{5}, v_{6}\right) .{ }^{12}$ Using Proposition 2, we can calculate this conditional probability distribution. To see why this is possible, observe that $Y_{A_{2}}$ is a linear function of two independent, normally distributed variables $\pi_{5}$ and $\pi_{6}$, and therefore it is distributed normally. Since $v_{5}$ and $v_{6}$ are also normally distributed variables, Proposition 2 applies. Let

$$
Z=\left(\begin{array}{c}
Y_{A_{2}} \\
v_{5} \\
v_{6}
\end{array}\right)
$$

Note that $Z$ is distributed as

$$
Z=\left(\begin{array}{c}
Y_{A_{2}} \\
v_{5} \\
v_{6}
\end{array}\right) \sim N\left(\left(\begin{array}{c}
U_{6}-U_{5} \\
0 \\
0
\end{array}\right),\left(\begin{array}{ccc}
2 & -\rho & \rho \\
-\rho & 1 & 0 \\
\rho & 0 & 1
\end{array}\right)\right) .
$$

From Proposition 2, we can calculate the mean and the variance of this conditional distri- 
bution as

$$
\begin{aligned}
E\left[Y_{A_{2}} \mid v_{5}, v_{6}\right] & =U_{6}-U_{5}+(-\rho, \rho)\left(\begin{array}{l}
v_{5} \\
v_{6}
\end{array}\right) \\
& =U_{6}-U_{5}+\rho\left(v_{6}-v_{5}\right) \\
\operatorname{Var}\left[Y_{A_{2}} \mid v_{5}, v_{6}\right] & =2-(-\rho, \rho)\left(\begin{array}{c}
-\rho \\
\rho
\end{array}\right) \\
& =2-2 \rho^{2}
\end{aligned}
$$

Hence,

$$
\begin{aligned}
p_{6}^{A_{1}} & =\operatorname{Pr}\left(Y_{A_{2}} \geq 0 \mid v_{5}, v_{6}\right) \\
& =\Phi\left(\frac{E\left[Y_{A_{2}} \mid v_{5}, v_{6}\right]}{\sqrt{\operatorname{Var}\left[Y_{A_{2}} \mid v_{5}, v_{6}\right]}}\right) \\
& =\Phi\left(\frac{U_{6}-U_{5}+\rho\left(v_{6}-v_{5}\right)}{\sqrt{2-2 \rho^{2}}}\right)
\end{aligned}
$$

To find $p_{2}$, we need to find $\operatorname{Pr}\left(Y_{A_{1}} \geq 0\right)$. This is a bit more involved than finding $p_{4}$, because unlike $Y_{B}$, we cannot define $Y_{A_{1}}$ as a linear function of independent normally distributed random variables $v_{1}, v_{3}, v_{5}$, and $v_{6}$. This is due to the probability expression $p_{6}^{A_{1}}$ that appears in $Y_{A_{1}}$ as a non-linear function of $v_{5}$ and $v_{6}$. In particular, $p_{6}^{A_{1}}$ is a function of $v_{6}-v_{5}$.

We instead calculate this probability without deriving the exact distribution of $Y_{A_{1}}$. We use two short-cuts. First, note that we can express the probability of an event as the expectation of the indicator variable for that event. In other words, if we define a random variable $I_{Y}$ as 


$$
I_{Y}= \begin{cases}1 & \text { if } Y_{A_{1}} \geq 0 \\ 0 & \text { otherwise }\end{cases}
$$

Then, $\operatorname{Pr}\left(Y_{A_{1}} \geq 0\right)$ becomes

$$
\operatorname{Pr}\left(Y_{A_{1}} \geq 0\right)=E\left[I_{Y}\right]
$$

Our second short-cut involves eliminating the $v_{6}-v_{5}$ term in $p_{6}^{A_{1}}$ that appears in $\operatorname{Pr}\left(Y_{A_{1}} \geq\right.$ $0)$. We will do that by conditioning on $v_{6}-v_{5}$. Intuitively, this means that if we knew $v_{6}-v_{5}$, then calculating $\operatorname{Pr}\left(Y_{A_{1}} \geq 0\right)$ would no longer be a problem because $Y_{A_{1}}$ would just become a linear function of normally distributed independent variables $v_{1}, v_{3}, v_{5}$, and $v_{6}$. To achieve this, we will use equality 38 and the Law of Iterated Expectations (LIE). For ease of presentation, we define $\epsilon=v_{6}-v_{5}$. Using LIE, we can rewrite the right-hand side of equation 38 as

$$
E\left[I_{Y}\right]=E_{\epsilon}\left[E\left[I_{Y} \mid \epsilon\right]\right]
$$

Also, using the fact that the conditional probability of an event is equal to the conditional expectation of the indicator variable of that event, we can replace the right-hand side with the following: 


$$
\begin{aligned}
E\left[I_{Y}\right] & =E_{\epsilon}\left[E\left[I_{Y} \mid \epsilon\right]\right] \\
& =E_{\epsilon}\left[\operatorname{Pr}\left(Y_{A_{1}} \geq 0 \mid \epsilon\right)\right]
\end{aligned}
$$

Note that if we knew $\epsilon=v_{6}-v_{5}$, then $Y_{A_{1}}$ would just become a linear function of normally distributed independent variables. Call this variable $Y_{A_{1}}^{*}$ to differentiate it from $Y_{A_{1}} \cdot Y_{A_{1}}^{*}$ is normally distributed with

$$
\begin{aligned}
E\left[Y_{A_{1}}^{*}\right] & =\left(1-p_{4}\right) U_{3}+p_{4}\left(1-\bar{p}_{6}^{A_{1}}\right) U_{5}+p_{4} \bar{p}_{6}^{A_{1}} U_{6}-U_{1} \\
\operatorname{Var}\left[Y_{A_{1}}^{*}\right] & =\left(1-p_{4}\right)^{2}+p_{4}^{2}\left(1-\bar{p}_{6}^{A_{1}}\right)^{2}+p_{4}^{2}\left(\bar{p}_{6}^{A_{1}}\right)^{2}+1
\end{aligned}
$$

We use $\bar{p}_{6}^{A_{1}}$ instead of $p_{6}^{A_{1}}$ to emphasize that $\bar{p}_{6}^{A_{1}}$ is just a constant when we know $\epsilon$. We can rewrite Equation 41 as

$$
\begin{aligned}
E\left[I_{Y}\right] & =E_{\epsilon}\left[\operatorname{Pr}\left(Y_{A_{1}} \geq 0 \mid \epsilon\right)\right] \\
& =E_{\epsilon}\left[\operatorname{Pr}\left(Y_{A_{1}}^{*} \geq 0 \mid \epsilon\right)\right]
\end{aligned}
$$

Hence, within the expectation, we now have the probability of a normally distributed variable conditional on another normally distributed variable. We can easily use Proposition 2 to calculate this probability. Define

$$
Z^{*}=\left(\begin{array}{c}
Y_{A_{1}}^{*} \\
\epsilon
\end{array}\right)
$$


Note that $Z^{*}$ is distributed as

$$
N\left(\left(\begin{array}{cc}
\left(1-p_{4}\right) U_{3}+p_{4}\left(1-\bar{p}_{6}^{A_{1}}\right) U_{5}+p_{4} \bar{p}_{6}^{A_{1}} U_{6}-U_{1} \\
0
\end{array}\right),\left(\begin{array}{cc}
\left(1-p_{4}\right)^{2}+p_{4}^{2}\left(1-\bar{p}_{6}^{A_{1}}\right)^{2}+p_{4}^{2}\left(\bar{p}_{6}^{A_{1}}\right)^{2}+1 & p_{4}\left(2 \bar{p}_{6}^{A_{1}}-1\right) \\
p_{4}\left(2 \bar{p}_{6}^{A_{1}}-1\right) & 2
\end{array}\right)\right)
$$

From this bivariate normal distribution, we calculate the mean and the variance of the conditional distribution specified within the expectation argument in Equation 45 as:

$$
\begin{aligned}
E\left[Y_{A_{1}}^{*} \mid \epsilon\right] & =\left(1-p_{4}\right) U_{3}+p_{4}\left[\left(1-\bar{p}_{6}^{A_{1}}\right) U_{5}+\bar{p}_{6}^{A_{1}} U_{6}\right]-U_{1}+\frac{p_{4}\left[2 \bar{p}_{6}^{A_{1}}-1\right] \epsilon}{2} \\
\operatorname{Var}\left[Y_{A_{1}}^{*} \mid \epsilon\right] & =\left(1-p_{4}\right)^{2}+\frac{p_{4}^{2}}{2}+1 .
\end{aligned}
$$

We can now easily calculate the probability within the expectation in Equation 45 as

$$
\operatorname{Pr}\left(Y_{A_{1}}^{*} \geq 0 \mid \epsilon\right)=\Phi\left(\frac{\left(1-p_{4}\right) U_{3}+p_{4}\left[\left(1-\bar{p}_{6}^{A_{1}}\right) U_{5}+\bar{p}_{6}^{A_{1}} U_{6}\right]-U_{1}+\frac{p_{4}\left[2 \bar{p}_{6}^{A_{1}}-1\right] \epsilon}{2}}{\sqrt{\left(1-p_{4}\right)^{2}+\frac{p_{4}^{2}}{2}+1}}\right)
$$

Thus, finally, $p_{2}$ is equal to

$$
p_{2}=E_{\epsilon}\left[I_{Y}\right]=E_{\epsilon}\left[\Phi\left(\frac{\left(1-p_{4}\right) U_{3}+p_{4}\left[\left(1-\bar{p}_{6}^{A_{1}}\right) U_{5}+\bar{p}_{6}^{A_{1}} U_{6}\right]-U_{1}+\frac{p_{4}\left[2 \bar{p}_{6}^{A_{1}}-1\right] \epsilon}{2}}{\sqrt{\left(1-p_{4}\right)^{2}+\frac{p_{4}^{2}}{2}+1}}\right)\right]
$$

Finally, we calculate $p_{6}^{B}$, the probability that Player $A_{2}$ chooses action $a_{6}$ given that Player $A_{2}$ has chosen action $a_{2}$. Note that

$$
\begin{aligned}
p_{6}^{B} & =\operatorname{Pr}\left(Y_{A_{2}} \geq 0 \mid Y_{A_{1}} \geq 0\right) \\
& =\frac{\operatorname{Pr}\left(Y_{A_{2}} \geq 0, Y_{A_{1}} \geq 0\right)}{p_{2}} .
\end{aligned}
$$


To characterize $\operatorname{Pr}\left(Y_{A_{2}} \geq 0, Y_{A_{1}} \geq 0\right)$, we make the following claim:

Claim 3 Given $\epsilon, Y_{A_{1}}^{*}$ and $Y_{A_{2}}$ are independent. That is,

$$
\operatorname{Cov}\left[Y_{A_{1}}^{*}, Y_{A_{2}} \mid \epsilon\right]=0
$$

Proof We already showed that $Y_{A_{1}}^{*}, Y_{A_{2}}$, and $\epsilon$ are all normally distributed variables, as they are linear functions of independent normally distributed variables. We can again use Proposition 2 to derive the joint distribution of $Y_{A_{1}}^{*}$ and $Y_{A_{2}}$ conditional on $\epsilon$.

We first define a random vector $\Gamma$ from a multivariate normal distribution:

$$
\Gamma=\left(\begin{array}{c}
Y_{A_{1}}^{*} \\
Y_{A_{2}} \\
\epsilon
\end{array}\right)
$$

Then, $E[\Gamma]$ and $\operatorname{Var}[\Gamma]$ are as follows:

$$
\begin{aligned}
E[\Gamma]= & \left(\begin{array}{c}
E\left[Y_{A_{1}}^{*}\right] \\
E\left[Y_{A_{2}}\right] \\
E[\epsilon]
\end{array}\right) \\
= & \left(\begin{array}{c}
\left(1-p_{4}\right) U_{3}+p_{4}\left(1-\bar{p}_{6}^{A_{1}}\right) U_{5}+p_{4} \bar{p}_{6}^{A_{1}} U_{6}-U_{1} \\
U_{6}-U_{5} \\
0
\end{array}\right) \\
\operatorname{Var}[\Gamma]= & \left(\begin{array}{c}
\left.\left(1-p_{4}\right)^{2}+p_{4}^{2}\left(1-\bar{p}_{6}^{A_{1}}\right)^{2}+p_{4}^{2}\left(\bar{p}_{6}^{A_{1}}\right)^{2}+1 \bar{p}_{6}^{A_{1}}-1\right) \\
p_{4} \rho\left(2 \bar{p}_{6}^{A_{1}}-1\right) \\
p_{4}\left(2 \bar{p}_{6}^{A_{1}}-1\right) \\
2 \\
2 \rho
\end{array}\right)
\end{aligned}
$$


From Proposition 2, we get the covariance of $Y_{A_{1}}^{*}$ and $Y_{A_{2}}$ given $\epsilon$ as

$$
\begin{aligned}
\operatorname{Cov}\left[Y_{A_{1}}^{*}, Y_{A_{2}} \mid \epsilon\right] & =\operatorname{Var}[\Gamma]_{12}-\operatorname{Var}[\Gamma]_{13} \operatorname{Var}[\Gamma]_{32} / \operatorname{Var}[\Gamma]_{33} \\
& =p_{4} \rho\left(2 \bar{p}_{6}^{A_{1}}-1\right)-p_{4}\left(2 \bar{p}_{6}^{A_{1}}-1\right) 2 \rho / 2 \\
& =0 .
\end{aligned}
$$

Hence, given $\epsilon, Y_{A_{1}}^{*}$ and $Y_{A_{2}}$ are independent.

We can now write $\operatorname{Pr}\left(Y_{A_{2}} \geq 0, Y_{A_{1}} \geq 0\right)$ as

$$
\begin{aligned}
\operatorname{Pr}\left(Y_{A_{2}} \geq 0, Y_{A_{1}} \geq 0\right) & =E_{\epsilon}\left[\operatorname{Pr}\left(Y_{A_{2}} \geq 0, Y_{A_{1}} \geq 0 \mid \epsilon\right)\right] \\
& =E_{\epsilon}\left[\operatorname{Pr}\left(Y_{A_{2}} \geq 0, Y_{A_{1}}^{*} \geq 0 \mid \epsilon\right)\right] \\
& =E_{\epsilon}\left[\operatorname{Pr}\left(Y_{A_{2}} \geq 0 \mid \epsilon\right) \operatorname{Pr}\left(Y_{A_{1}}^{*} \geq 0 \mid \epsilon\right)\right]
\end{aligned}
$$

and $p_{6}^{B}$ as

$$
\begin{aligned}
p_{6}^{B} & =\operatorname{Pr}\left(Y_{A_{2}} \geq 0 \mid Y_{A_{1}} \geq 0\right) \\
& =\frac{\operatorname{Pr}\left(Y_{A_{2}} \geq 0, Y_{A_{1}} \geq 0\right)}{p_{2}} \\
& =\frac{E_{\epsilon}\left[\operatorname{Pr}\left(Y_{A_{2}} \geq 0 \mid \epsilon\right) \operatorname{Pr}\left(Y_{A_{1}}^{*} \geq 0 \mid \epsilon\right)\right]}{p_{2}} \\
& =\frac{E_{\epsilon}\left[\Phi\left(\frac{U_{6}-U_{5}+\rho \epsilon}{\sqrt{2-2 \rho^{2}}}\right) \Phi\left(\frac{\left(1-p_{4}\right) U_{3}+p_{4}\left[\left(1-\bar{p}_{6}^{A_{1}}\right) U_{5}+\bar{p}_{6}^{A_{1}} U_{6}\right]-U_{1}+\frac{p_{4}\left[2 \bar{p}_{6}^{A_{1}}-1\right] \epsilon}{2}}{\sqrt{\left(1-p_{4}\right)^{2}+\frac{p_{4}^{2}}{2}+1}}\right)\right]}{p_{2}}
\end{aligned}
$$




\section{A.2 Proofs of Claims 1 and 2}

\section{A.2.1 Equivalence of CAM and IAM when $\rho=0$.}

First, observe that when $\rho=0, p_{6}^{A_{1}}$ in equation 13 reduces to $\Phi\left(\frac{U_{6}-U_{5}}{\sqrt{2}}\right)$, which is equal to the IAM probability $p_{6}$ in equation 17 . Second, observe that when $\rho=0$, the first $\Phi()$ argument inside the expectation in equation 14 is no longer a function of $\epsilon$ and therefore can be taken out of the expectation. The remaining part of the expectation is equal to $p_{2}$ in equation 16 , and $p_{2}$ in the numerator and the denominator cancel each other out. Therefore, $p_{6}=p_{6}^{B}=p_{6}^{A_{1}}$ when $\rho=0$. Third, $p_{4}$ in equation 15 as a function of $p_{6}^{B}$ reduces to $p_{4}$ in equation 18 as $p_{6}=p_{6}^{B}$. Finally, in calculating $p_{2}$ when $\rho=0$, note that we no longer need to condition on $\epsilon=v_{6}-v_{5}$ and use the Law of Iterated Expectations, because $Y_{A_{1}}$ is now a linear function of independent normally distributed random variables and itself is normally distributed. This results in the probability $p_{2}$ as expressed in equation 19.

\section{A.2.2 Equivalence of CAM and SAM when $\rho=1$.}

In this section, we show that CAM probabilities converge to SAM probabilities given in Claim 2 when $\rho \rightarrow 1$. Define an indicator function $I_{6}$ that takes the value 1 when $\max \left\{U_{6}+\right.$ $\left.v_{6}, U_{5}+v_{5}\right\}=U_{6}+v_{6}$ and 0 otherwise. Thus, in the last node, $A_{2}$ chooses $a_{6}$ if and only if $I_{6}=1$. Also, since $\rho=1$, define $\epsilon=v_{6}-v_{5}=\pi_{6}-\pi_{5}$. Thus, when $\rho=1, p_{6}^{A}$ in CAM converges to $I_{6}$.

Observe that, when $\rho=1$, derivation of $p_{4}$ follows the identical steps as in the more general CAM case as defined in equation 28. Moreover, $A_{1}$ knows exactly what $A_{2}$ will do at the last decision node due to the perfect correlation. Thus, $A_{1}$ chooses $a_{2}$ if and only if

$$
\left(1-p_{4}\right)\left(U_{3}+v_{3}\right)+p_{4} \max \left\{U_{6}+v_{6}, U_{5}+v_{5}\right\} \geq U_{1}+v_{1}
$$


Thus, one way to derive $p_{2}$ is as follows:

$$
\begin{aligned}
p_{2} & =\operatorname{Pr}\left(\max \left\{U_{6}+v_{6}, U_{5}+v_{5}\right\} \geq \frac{U_{1}+v_{1}-\left(1-p_{4}\right)\left(U_{3}+v_{3}\right)}{p_{4}}\right) \\
& =1-\operatorname{Pr}\left(U_{6}+v_{6}<\frac{U_{1}+v_{1}-\left(1-p_{4}\right)\left(U_{3}+v_{3}\right)}{p_{4}} \wedge U_{5}+v_{5}<\frac{\left.U_{1}+v_{1}-\left(1-p_{4}\right)\left(U_{3}+v_{3}\right)_{71}\right)}{p_{4}}\right) \\
& =1-\Phi_{2}\left[\frac{U_{1}-\left(1-p_{4}\right) U_{3}-p_{4} U_{5}}{\sqrt{p_{4}^{2}+1+\left(1-p_{4}\right)^{2}}}, \frac{U_{1}-\left(1-p_{4}\right) U_{3}-p_{4} U_{6}}{\sqrt{p_{4}^{2}+1+\left(1-p_{4}\right)^{2}}}, \frac{1+\left(1-p_{4}\right)^{2}}{p_{4}^{2}+1+\left(1-p_{4}\right)^{2}}\right]
\end{aligned}
$$

Equivalently, we can calculate this probability by conditioning on $\epsilon$ and then calculating the conditional probability using Proposition 2:

$$
\begin{aligned}
p_{2} & =E_{\epsilon}\left[\operatorname{Pr}\left(\max \left\{U_{6}+v_{6}, U_{5}+v_{5}\right\} \geq \frac{U_{1}+v_{1}-\left(1-p_{4}\right)\left(U_{3}+v_{3}\right)}{p_{4}} \mid \epsilon\right)\right] \\
& =E_{\epsilon}\left[\Phi\left(\frac{\left(1-p_{4}\right) U_{3}+p_{4}\left[\left(1-I_{6}\right) U_{5}+I_{6} U_{6}\right]-U_{1}+\frac{p_{4}\left[2 I_{6}-1\right] \epsilon}{2}}{\sqrt{\left(1-p_{4}\right)^{2}+\frac{p_{4}^{2}}{2}+1}}\right)\right]
\end{aligned}
$$

which is also $p_{2}$ for CAM when $\rho=1$.

Finally, we can calculate $p_{6}^{B}$ as

$$
\begin{aligned}
p_{6}^{B} & =\operatorname{Pr}\left(Y_{A_{2}} \geq 0 \mid Y_{A_{1}} \geq 0\right) \\
& =\frac{\operatorname{Pr}\left(Y_{A_{2}} \geq 0, Y_{A_{1}} \geq 0\right)}{p_{2}} \\
& \left.=\frac{\operatorname{Pr}\left(U_{6}+v_{6} \geq U_{5}+v_{5},\left(1-p_{4}\right)\left(U_{3}+v_{3}\right)+p_{4} \max \left\{U_{6}+v_{6}, U_{5}+v_{5}\right\} \geq U_{1}+(77)\right.}{p_{2}}\right] \\
& =\Phi_{2}\left[\frac{U_{6}-U_{5}}{\sqrt{2}}, \frac{p_{4} U_{6}-U_{1}+\left(1-p_{4}\right) U_{3}}{\sqrt{p_{4}^{2}+1+\left(1-p_{4}\right)^{2}}}, \frac{p_{4}}{\sqrt{2\left(1+\left(1-p_{4}\right)^{2}+p_{4}^{2}\right)}}\right] / p_{2}
\end{aligned}
$$

Equivalently, we can calculate this probability by again conditioning on $\epsilon$ and then calculating the conditional probability $\operatorname{Pr}\left(Y_{A_{2}} \geq 0, Y_{A_{1}} \geq 0 \mid \epsilon\right)$ using Proposition 2. Conditioning on 
$\epsilon$ allows us to use Claim 3 to treat $\operatorname{Pr}\left(Y_{A_{2}} \geq 0 \mid \epsilon\right)$ and $\operatorname{Pr}\left(Y_{A_{1}} \geq 0 \mid \epsilon\right)$ as probabilities of two independent events. Hence,

$$
\begin{aligned}
p_{6}^{B} & =\frac{E_{\epsilon}\left[\operatorname{Pr}\left(Y_{A_{2}} \geq 0, Y_{A_{1}} \geq 0 \mid \epsilon\right)\right]}{p_{2}} \\
& =\frac{E_{\epsilon}\left[\operatorname{Pr}\left(Y_{A_{2}} \geq 0 \mid \epsilon\right) \operatorname{Pr}\left(Y_{A_{1}} \geq 0 \mid \epsilon\right)\right]}{p_{2}} \\
& =\frac{E_{\epsilon}\left[I_{6} \Phi\left(\frac{\left(1-p_{4}\right) U_{3}+p_{4}\left[\left(1-I_{6}\right) U_{5}+I_{6} U_{6}\right]-U_{1}+\frac{p_{4}\left[2 I_{6}-1\right] \epsilon}{2}}{\sqrt{\left(1-p_{4}\right)^{2}+\frac{p_{4}^{2}}{2}+1}}\right)\right]}{p_{2}}
\end{aligned}
$$

which is identical to $p_{6}^{B}$ for CAM when $\rho=1$.

\section{B Monte Carlo Analysis}

\section{B.1 MC Analysis 1: Consistency of CAM}

In this section, we evaluate the unbiasedness and consistency of estimates provided by CAM. In terms of specifying the outcome utilities for the analysis, we look at two scenarios. In the first scenario, we select the parameter values such that the amount of belief updating by B about $A_{2}$ 's choice is expected to be minimal. In the second setting, we present a case where we expect a larger amount of belief updating by B. Parameter values for the two scenarios are presented in Table 4 . What makes the second scenario permit more updating than the first? First, player A does not have much to gain by choosing $a_{2}$ rather than $a_{1}$ in the second setting because $U_{A, 3}$, A's payoff when player B chooses $a_{3}$, is only 0.5 , while this value is 1 in the first case. Second, the second scenario indicates that A incurs substantial losses 
when $\mathrm{B}$ chooses $a_{4}$, because $U_{A, 5}$ and $U_{A, 6}$ are -4 and -5 respectively, whereas they are only -0.5 and -1 in the first setting. Third, the second specification gives B a relatively strong incentive to choose $a_{4}$ because $U_{B, 5}$ is much higher in the second case (5) than the first (1). Consequently, the second scenario implies a situation where, on average, player A has less incentive to choose $a_{2}$ over $a_{1}$ and player $\mathrm{B}$ has more incentive to choose $a_{4}$ over $a_{3}$. If player A chooses $a_{2}$, this implies that the private components of $U_{A, 1}, U_{A, 3}$, and $U_{A, 5}$ are likely to be larger than expected a priori, and this causes Player B to update her beliefs by a larger amount after observing $a_{2}$.

[Table 4 about here.]

In addition to two configurations described above, we consider three correlation values $\rho \in\{0, .5, .9\}$ and four sample sizes $N \in\{250,500,1000,5000\}$. For each parameter and sample size combination, $1000 \mathrm{MC}$ iterations are completed, which give us the approximate sampling distributions of the CAM estimates. ${ }^{13}$

We estimate $U_{A, 1}, U_{A, 5}, U_{A, 6}, U_{B, 3}$, and $\rho$ directly. The remaining three mean payoffs, $U_{A, 3}, U_{B, 5}$, and $U_{B, 6}$, are assumed to be a linear combination of regressors:

$$
\begin{aligned}
& U_{A, 3}=X_{A, 3} \beta_{A, 3} \\
& U_{B, 5}=X_{B, 5} \beta_{B, 5} \\
& U_{B, 6}=X_{B, 6} \beta_{B, 6},
\end{aligned}
$$

where $X_{A, 3}, X_{B, 5}$, and $X_{B, 6}$ are regressors randomly drawn from a standard normal distribution. Thus, in each iteration of the MC analysis, we also estimate $\hat{\beta}_{A, 3}, \hat{\beta}_{B, 5}$, and 
$\hat{\beta}_{B, 6}$.

Table 5 reports the mean coefficient estimates. The top half of the table is for coefficients from the small updating scenario, and the bottom half is for a large updating case. Using the same structure, Table 6 reports the root mean squared error (RMSE) of the estimated coefficients: $\sqrt{E(\hat{\theta}-\theta)^{2}}$, where $\theta$ denotes the true parameter value from the data generating process, and $\hat{\theta}$ is the CAM parameter estimate. RMSE is the square root of the mean squared error (MSE). MSE of an estimate is the sum of the variance and the squared bias of that estimate, and in that sense, smaller MSE and RMSE values indicate a more precise estimator. We chose to report RMSE, because it has the advantage of being on the same scale as the estimated parameter.

In both small and large updating settings, all coefficients on average are estimated very close to the true parameter values, and the accuracy of the estimates increases as the sample size increases. For positive $\rho$, there seems to be a downward bias in small samples, which disappears as the samples get larger. RMSE values also indicate that the precision of the estimates get significantly better as the sample size increases. RMSE values approach zero as the samples get larger, which means that any potential bias in the estimates disappear, and the variance of the estimates approach zero, indicating consistency.

[Table 5 about here.]

[Table 6 about here.]

The MC analysis in this section has shown that CAM can be estimated in a variety of parameter configurations and the estimates are unbiased and consistent. The analysis did not determine, however, whether CAM has an advantage over SAM or IAM in providing correct inferences. The next section provides such a comparison by evaluating the performances of CAM, SAM, and IAM for different correlation values. 


\section{B.2 MC Analysis 2: Comparison of CAM, SAM, and IAM}

When should CAM be chosen over SAM or IAM? Are there cases in which the latter two should be preferred? This section provides an answer to these questions by comparing the three estimators across different correlation values. In this experiment, we set the parameter values to $U_{A, 1}=0, U_{A, 5}=-4, U_{A, 6}=-4.5, U_{B, 3}=0$. The remaining three observable payoff components, $U_{A, 3}, U_{B, 5}$, and $U_{B, 6}$ are assumed, as in the previous section, to be a linear combination of regressors:

$$
\begin{aligned}
& U_{A, 3}=X_{A, 3} \beta_{A, 3} \\
& U_{B, 5}=X_{B, 5} \beta_{B, 5} \\
& U_{B, 6}=X_{B, 6} \beta_{B, 6},
\end{aligned}
$$

where $X_{A, 3}, X_{B, 5}$, and $X_{B, 6}$ are randomly drawn from a standard normal distribution. We set $\beta_{A, 3}=6, \beta_{B, 5}=4$, and $\beta_{B, 6}=-6$. We chose this parameter configuration because it permits significant belief updating by B. In addition, we consider $\rho \in\{.05, .35, .65, .95\}$. $\rho=.05$ represents a scenario where the data-generating process is closer to IAM, and when $\rho=.95$, the process approximates SAM.

Figure 4 plots CAM equilibrium action probabilities $p_{2}, p_{4}$, and $p_{6}^{B}$ as a function of $\rho$, based on the data-generating process values for the outcome utilities. As the plot makes clear, the action probabilities are not linear and can be non-monotonic in $\rho$. More strikingly, depending on the value of $\rho$, action probabilities can shift from being very close to zero to .40, which makes correctly estimating $\rho$ a very important factor for achieving unbiased 
inferences.

[Figure 4 about here.]

Based on each possible combination of parameters specified above, we generated 1000 different samples of size $\mathrm{N}=10000$. Having a larger sample size makes standard errors of the estimated coefficients smaller and makes it possible to focus mainly on the potential bias in the estimates. In each of the 1000 samples generated, we estimated CAM, SAM, and IAM to approximate the sampling distributions of the seven outcome utility parameter estimates. CAM also included an additional parameter $\rho$ to be estimated.

Table 7 presents the means of estimated parameters. As in the previous MC analysis, CAM on average successfully captures all the parameter values. IAM, on the other hand, only produces unbiased estimates when $\rho$ is .05, but fails to do so for larger $\rho$. Similarly, SAM fails to provide unbiased estimates for most of the coefficient values, even when $\rho=.95$.

By themselves, the results presented in Table 7 do not provide enough information to indicate CAM as a better choice over the other two estimators in terms of making inferences. For one thing, the estimated coefficients in IAM and SAM might be calculated on a different scale than the CAM estimates due to the differences in the variance-covariance structure. Thus, it is possible that IAM and SAM coefficients are not actually biased, but rather scaled differently. Action and outcome probabilities do not suffer from this scaling issue. Second, in empirical analyses, scholars are more often interested in the action or outcome probability estimates than in the exact coefficient estimates. Thus, if IAM and SAM provide unbiased estimates of those probabilities for different correlation values, the usefulness of CAM would be limited. For these reasons, Table 8 compares the three estimators based on the action probability estimates they produce for each player, and how closely these estimates approximate the probabilities specified by the data-generating process (DGP). The table provides the mean estimates for $p_{2}, p_{4}, p_{6}^{B}$, and $p_{6}$ as well as their RMSE values. ${ }^{14}$ 
In all four correlation values considered, CAM on average provides very accurate estimates of the action probabilities. Except when $\rho=.05$, IAM provides biased probability estimates, with bias as large as .25 when $\rho=.65$. Although not as biased as IAM, SAM also provides biased probability estimates, with bias as much as .08 for $p_{4}$ when $\rho=.65$. Perhaps more interestingly, CAM does better than SAM even when $\rho=.95$. RMSE values also produce similar implications. Given the large sample size, a large RMSE is mainly an indication of bias rather than the variance of the estimator. Both IAM and SAM produce RMSE values significantly larger than CAM, except when $\rho=.05$ for IAM.

The main conclusion from this analysis is that, when the underlying correlation value is unknown, CAM is clearly the appropriate choice of estimator over SAM or IAM, as it provides unbiased utility and probability estimators without making a priori parametric assumptions about $\rho$. Even when the correlation is suspected to be very small, or very close to one, CAM fares at least as well as IAM and SAM. Given that it is very unlikely for a scholar to know the approximate value of $\rho$ without estimating it, our results suggest that CAM should be preferred over the two other alternatives when the structure of the interaction indicates a potential correlation between private components of the two actors.

[Table 7 about here.]

[Table 8 about here.] 


\begin{tabular}{|c|c|c|}
\hline Variables & Utilities & Measures \\
\hline MaxAge & $S Q_{A}$ & The log of maximum age of the states in the dyad \\
\hline Alliance & $A C Q_{A}$ and $A C Q_{B}$ & Tau-b score using alliances in the dyad's region \\
\hline CivilWar $_{B}$ & $A C Q_{A}$ and $A C Q_{B}$ & Dummy: was B involved in a civil war? \\
\hline Contiguous & $A C Q_{A}$ and $A C Q_{B}$ & $\begin{array}{l}\text { Dummy: do states share a land border or are } \\
\text { separated by less than } 150 \text { miles of water? }\end{array}$ \\
\hline Democracy $_{A}$ & $B D_{A}, S F_{A}$, and $\rho$ & Dummy: was A democratic? \\
\hline CapShare $_{A}$ & $S F_{A}$ and $S F_{B}$ & A's share of capabilities in the dyad \\
\hline Develop $_{A}$ & $S F_{A}$ & The log of energy consumption per capita for $A$ \\
\hline Democracy $_{B}$ & $S F_{B}$ & Dummy: was B democratic? \\
\hline Develop $_{B}$ & $S F_{B}$ & The log of energy consumption per capita for $B$ \\
\hline Population $_{A}$ & $\rho$ & The log of total population of $\mathrm{A}$ \\
\hline
\end{tabular}

Table 1: Variables of Lewis and Schultz (2005) and CAM Application The source of Democracy $A$ and Democracy De $_{B}$ Polity IV data set and the rest of variables is found from Correlates of War data set. All variables including Population can $_{\text {ca }}$ be obtained using EUGene (http://www.eugenesoftware.org). 


\begin{tabular}{|c|c|c|c|c|c|c|}
\hline \multicolumn{3}{|c|}{ Estimation model: } & \multicolumn{2}{|c|}{ SAM } & \multicolumn{2}{|c|}{ CAM1 } \\
\hline & & & Est. & $\mathrm{SE}$ & Est. & $\mathrm{SE}$ \\
\hline \multirow[t]{2}{*}{ SQ } & $S Q_{A}$ & Constant & 0 & & 0 & \\
\hline & & MaxAge & $0.12^{*}$ & $(0.07)$ & $0.23^{* *}$ & $(0.09)$ \\
\hline \multirow[t]{8}{*}{$\mathrm{ACQ}$} & $A C Q_{A}$ & Constant & 1.77 & $(1.27)$ & 0.76 & $(2.46)$ \\
\hline & & Alliance & -1.18 & $(0.89)$ & $-3.51^{*}$ & $(2.06)$ \\
\hline & & CivilWar $_{B}$ & 1.07 & $(1.16)$ & $5.43^{*}$ & $(3.24)$ \\
\hline & & Contiguous & 0.72 & $(0.54)$ & 1.33 & $(0.98)$ \\
\hline & $A C Q_{B}$ & Constant & -1.41 & $(1.27)$ & -1.19 & $(1.47)$ \\
\hline & & Alliance & 0.19 & $(0.89)$ & $1.06^{*}$ & $(0.59)$ \\
\hline & & CivilWar $_{B}$ & 0.15 & $(1.16)$ & -0.25 & $(0.42)$ \\
\hline & & Contiguous & -0.09 & $(0.54)$ & -0.08 & $(0.32)$ \\
\hline \multirow[t]{3}{*}{$\mathrm{BD}$} & $B D_{A}$ & Constant & $-4.06^{* *}$ & $(1.08)$ & $-5.47 * *$ & $(1.92)$ \\
\hline & & Democracy $_{A}$ & $-0.76^{* *}$ & $(0.33)$ & $-1.20^{* *}$ & $(0.60)$ \\
\hline & $B D_{B}$ & Constant & 0 & & 0 & \\
\hline \multirow[t]{9}{*}{$\overline{\mathrm{SF}}$} & $S F_{A}$ & Constant & $-3.62^{* *}$ & $(1.08)$ & $-4.94^{* *}$ & $(1.92)$ \\
\hline & & CapShare $_{A}$ & $0.83^{* *}$ & $(0.31)$ & $1.83^{* *}$ & $(0.88)$ \\
\hline & & Democracy $_{A}$ & $-0.79 * *$ & $(0.3)$ & $-1.35^{* *}$ & $(0.42)$ \\
\hline & & Develop $_{A}$ & $0.02 *$ & $(0.01)$ & 0.06 & $(0.05)$ \\
\hline & $S F_{B}$ & Constant & -1.97 & $(1.96)$ & -1.76 & $(2.10)$ \\
\hline & & CapShare $_{A}$ & 1.17 & $(0.77)$ & 1.87 & $(1.39)$ \\
\hline & & Democracy & 0.1 & $(0.09)$ & 0.28 & $(0.29)$ \\
\hline & & Develop $_{B}$ & -0.02 & $(0.02)$ & -0.05 & $(0.06)$ \\
\hline & $\rho$ & Constant & & & 1.31 & $(4.06)$ \\
\hline \multicolumn{3}{|l|}{$\mathrm{N}$} & \multicolumn{2}{|c|}{2187} & \multicolumn{2}{|c|}{2187} \\
\hline \multicolumn{3}{|c|}{ Log likelihood } & \multicolumn{2}{|c|}{-418.90} & \multicolumn{2}{|c|}{-416.64} \\
\hline \multicolumn{7}{|c|}{$* *=p<.05, *=.05<p<.1$ (two-tailed) } \\
\hline
\end{tabular}

Table 2: SAM and CAM Results on Lewis and Schultz (2005) Data Set (First Part) 


\begin{tabular}{|c|c|c|c|c|c|c|}
\hline \multicolumn{3}{|c|}{ Estimation model: } & \multicolumn{2}{|c|}{ CAM2 } & \multicolumn{2}{|c|}{ CAM3 } \\
\hline & & & Est. & $\mathrm{SE}$ & Est. & $\mathrm{SE}$ \\
\hline \multirow[t]{2}{*}{ SQ } & $S Q_{A}$ & Constant & 0 & & 0 & \\
\hline & & MaxAge & $0.38^{* *}$ & $(0.13)$ & $0.55^{* *}$ & $(0.13)$ \\
\hline \multirow[t]{8}{*}{$\mathrm{ACQ}$} & $A C Q_{A}$ & Constant & 3.56 & $(7.68)$ & 3.09 & $(4.88)$ \\
\hline & & Alliance & -5.39 & $(4.12)$ & $-5.75^{*}$ & $(3.28)$ \\
\hline & & CivilWar $_{B}$ & 4.31 & $(2.89)$ & 7.27 & $(4.74)$ \\
\hline & & Contiguous & 4.16 & $(3.59)$ & $3.01^{*}$ & $(1.57)$ \\
\hline & $A C Q_{B}$ & Constant & -4.32 & $(6.53)$ & -4.38 & $(4.41)$ \\
\hline & & Alliance & 0.73 & $(0.64)$ & 1.07 & $(0.71)$ \\
\hline & & CivilWar $_{B}$ & -0.10 & $(0.29)$ & -0.56 & $(0.52)$ \\
\hline & & Contiguous & -0.58 & $(0.43)$ & -0.34 & $(0.26)$ \\
\hline \multirow[t]{3}{*}{$\mathrm{BD}$} & $B D_{A}$ & Constant & -8.26 & $(7.20)$ & -8.16 & $(5.52)$ \\
\hline & & Democracy $_{A}$ & $-1.77^{*}$ & $(1.09)$ & $-1.59 * *$ & $(0.56)$ \\
\hline & $B D_{B}$ & Constant & 0 & & 0 & \\
\hline \multirow[t]{11}{*}{$\mathrm{SF}$} & $S F_{A}$ & Constant & -7.42 & $(7.17)$ & -7.60 & $(5.50)$ \\
\hline & & CapShare $_{A}$ & $1.13^{* *}$ & $(0.25)$ & $0.31^{*}$ & $(0.18)$ \\
\hline & & Democracy $_{A}$ & $-1.76^{*}$ & $(0.99)$ & $-1.63^{* *}$ & $(0.54)$ \\
\hline & & Develop $_{A}$ & 0.01 & $(0.01)$ & 0.00 & $(0.01)$ \\
\hline & $S F_{B}$ & Constant & -5.90 & $(9.08)$ & -6.23 & $(6.17)$ \\
\hline & & CapShare $_{A}$ & 2.69 & $(4.05)$ & 1.17 & $(1.12)$ \\
\hline & & Democracy $_{B}$ & 0.13 & $(0.24)$ & 0.14 & $(0.20)$ \\
\hline & & Develop $_{B}$ & 0.00 & $(0.02)$ & 0.00 & $(0.02)$ \\
\hline & $\rho$ & Constant & 0.53 & $(0.38)$ & $0.78^{* *}$ & $(0.25)$ \\
\hline & & Population $_{A}$ & $0.51^{*}$ & $(0.27)$ & $1.15^{* *}$ & $(0.21)$ \\
\hline & & Democracy $_{A}$ & & & 0.62 & $(0.46)$ \\
\hline \multicolumn{3}{|l|}{$\mathrm{N}$} & \multicolumn{2}{|c|}{2187} & \multicolumn{2}{|c|}{2187} \\
\hline \multicolumn{3}{|c|}{ Log likelihood } & \multicolumn{2}{|c|}{-399.81} & \multicolumn{2}{|c|}{-393.44} \\
\hline \multicolumn{7}{|c|}{$* *=p<.05, *=.05<p<.1$ (two-tailed) } \\
\hline
\end{tabular}

Table 3: SAM and CAM Results on Lewis and Schultz (2005) Data Set (Second Part) 
Difference between Independent Agent Model and Single Agent Model Big Small

\begin{tabular}{ccc} 
& (Large Updating) & (Small Updating) \\
\hline$U_{A, 1}$ & 0 & 0 \\
$U_{A, 3}$ & 0.5 & 1 \\
$U_{A, 5}$ & -4 & -0.5 \\
$U_{A, 6}$ & -5 & -1 \\
$U_{B, 3}$ & 0 & 0 \\
$U_{B, 5}$ & 5 & 1 \\
$U_{B, 6}$ & -1 & -1 \\
\hline$\rho$ & \multicolumn{2}{c}{$0,0.5$ and 0.9}
\end{tabular}

Table 4: True Parameter Specification 


\begin{tabular}{|c|c|c|c|c|c|c|c|c|c|}
\hline \multicolumn{10}{|c|}{$\begin{array}{l}\text { Parameter Setting 1: Small Updating } \\
\begin{array}{ll}\text { A's Utilities } & \text { B's Utilities }\end{array}\end{array}$} \\
\hline $\mathrm{N}$ & $U_{A, 1}$ & $U_{A, 3}$ & $U_{A, 5}$ & $U_{A, 6}$ & $U_{B, 3}$ & $U_{B, 5}$ & $U_{B, 6}$ & & ) \\
\hline & 0 & 1 & $-1 / 2$ & -1 & 0 & 1 & -1 & & \\
\hline 250 & 0.033 & 1.041 & -0.490 & -0.972 & 0.014 & 1.058 & -1.063 & 0.031 & $\rho=0$ \\
\hline 500 & 0.015 & 1.032 & -0.498 & -0.981 & 0.036 & 1.030 & -0.932 & 0.022 & \\
\hline 1000 & 0.011 & 1.009 & -0.500 & -0.981 & 0.027 & 1.015 & -0.953 & 0.003 & \\
\hline 5000 & 0.003 & 1.005 & -0.499 & -0.996 & 0.011 & 1.003 & -0.978 & -0.006 & \\
\hline 250 & -0.031 & 1.036 & -0.507 & -1.020 & -0.034 & 1.053 & -1.201 & 0.368 & $\rho=.5$ \\
\hline 500 & -0.025 & 1.011 & -0.502 & -1.005 & 0.003 & 1.026 & -1.040 & 0.393 & \\
\hline 1000 & -0.017 & 1.003 & -0.502 & -1.006 & 0.006 & 1.014 & -1.007 & 0.451 & \\
\hline 5000 & 0.003 & 1.003 & -0.500 & -1.000 & 0.004 & 1.006 & -0.994 & 0.503 & \\
\hline 250 & -0.031 & 1.031 & -0.513 & -1.006 & -0.015 & 1.045 & -1.115 & 0.766 & $\rho=.9$ \\
\hline 500 & -0.031 & 1.009 & -0.505 & -1.008 & -0.002 & 1.022 & -1.045 & 0.796 & \\
\hline 1000 & -0.015 & 1.011 & -0.502 & -1.002 & -0.001 & 1.008 & -1.003 & 0.843 & \\
\hline 5000 & -0.003 & 1.002 & -0.501 & -1.001 & 0.001 & 1.002 & -1.004 & 0.886 & \\
\hline
\end{tabular}

Parameter Setting 2: Large Updating

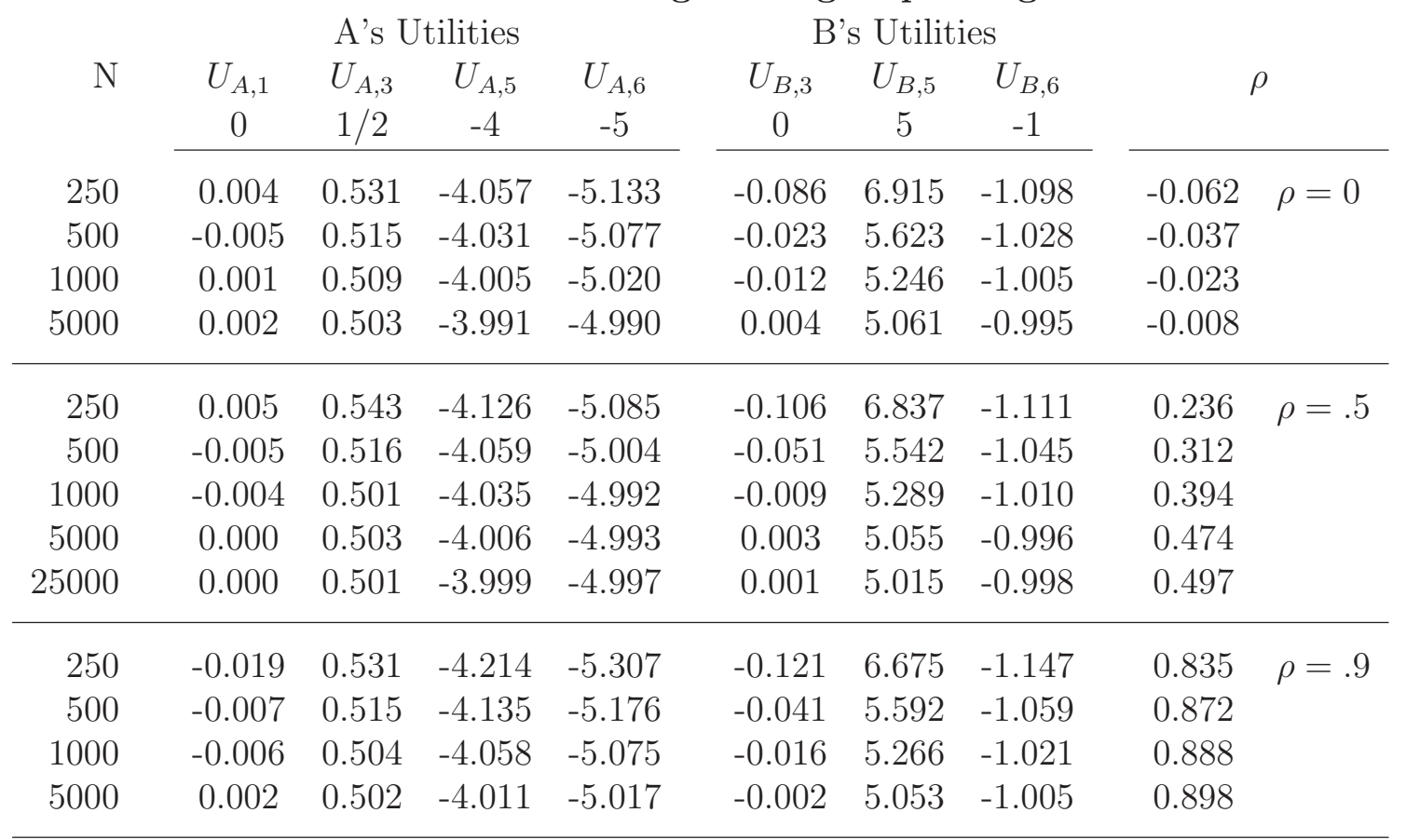

Table 5: Mean Parameter Estimates from CAM 


\begin{tabular}{|c|c|c|c|c|c|c|c|c|c|}
\hline \multirow{3}{*}{$\mathrm{N}$} & \multirow{2}{*}{\multicolumn{3}{|c|}{ Parameter }} & \multicolumn{4}{|c|}{$\begin{array}{c}\text { Setting 1: Small Updating } \\
\text { B's Utilities }\end{array}$} & \multirow{3}{*}{\multicolumn{2}{|c|}{$\rho$}} \\
\hline & $U_{A, 1}$ & & & $U_{A, 6}$ & $U_{B, 3}$ & $U_{B, 5}$ & $U_{B, 6}$ & & \\
\hline & 0 & 1 & $-1 / 2$ & -1 & 0 & 1 & -1 & & \\
\hline 250 & 0.226 & 0.260 & 0.168 & 0.258 & 0.488 & 0.246 & 1.261 & 0.588 & $\rho=0$ \\
\hline 500 & 0.174 & 0.174 & 0.118 & 0.188 & 0.279 & 0.177 & 0.671 & 0.494 & \\
\hline 1000 & 0.134 & 0.118 & 0.084 & 0.127 & 0.201 & 0.118 & 0.483 & 0.398 & \\
\hline 5000 & 0.069 & 0.054 & 0.035 & 0.054 & 0.087 & 0.051 & 0.208 & 0.209 & \\
\hline 250 & 0.223 & 0.257 & 0.164 & 0.247 & 0.471 & 0.250 & 1.220 & 0.565 & $\rho=.5$ \\
\hline 500 & 0.175 & 0.164 & 0.115 & 0.171 & 0.299 & 0.161 & 0.758 & 0.460 & \\
\hline 1000 & 0.132 & 0.117 & 0.079 & 0.118 & 0.198 & 0.117 & 0.486 & 0.361 & \\
\hline 5000 & 0.063 & 0.055 & 0.034 & 0.052 & 0.090 & 0.052 & 0.217 & 0.172 & \\
\hline 250 & 0.205 & 0.268 & 0.168 & 0.245 & 0.517 & 0.245 & 1.481 & 0.373 & $\rho=.9$ \\
\hline 500 & 0.145 & 0.171 & 0.113 & 0.163 & 0.315 & 0.172 & 0.801 & 0.305 & \\
\hline 1000 & 0.102 & 0.127 & 0.082 & 0.117 & 0.215 & 0.118 & 0.528 & 0.186 & \\
\hline 5000 & 0.041 & 0.053 & 0.035 & 0.050 & 0.095 & 0.051 & 0.231 & 0.064 & \\
\hline
\end{tabular}

\begin{tabular}{|c|c|c|c|c|c|c|c|c|c|}
\hline & & $\begin{array}{l}\text { Para } \\
\text { A's U }\end{array}$ & $\begin{array}{l}\text { meter } \\
\text { ilities }\end{array}$ & etti & $\begin{array}{r}\text { Larg } \\
\text { B }\end{array}$ & $\begin{array}{l}\text { e Upd } \\
\text { s Utilit }\end{array}$ & $\begin{array}{l}\text { ating } \\
\text { es }\end{array}$ & & \\
\hline $\mathrm{N}$ & $U_{A, 1}$ & $U_{A, 3}$ & $U_{A, 5}$ & $U_{A, 6}$ & $U_{B, 3}$ & $U_{B, 5}$ & $U_{B, 6}$ & & $\rho$ \\
\hline & 0 & $1 / 2$ & -4 & -5 & 0 & 5 & -1 & & \\
\hline 250 & 0.218 & 0.258 & 0.641 & 1.055 & 1.169 & 6.969 & 1.182 & 0.407 & $\rho=0$ \\
\hline 500 & 0.154 & 0.167 & 0.431 & 0.717 & 0.377 & 1.792 & 0.391 & 0.338 & \\
\hline 1000 & 0.111 & 0.112 & 0.295 & 0.495 & 0.213 & 0.857 & 0.236 & 0.284 & \\
\hline 5000 & 0.047 & 0.050 & 0.128 & 0.220 & 0.088 & 0.350 & 0.100 & 0.157 & \\
\hline 250 & 0.224 & 0.243 & 0.651 & 0.951 & 1.054 & 6.729 & 1.070 & 0.523 & $\rho=.5$ \\
\hline 500 & 0.159 & 0.165 & 0.431 & 0.603 & 0.411 & 1.778 & 0.423 & 0.401 & \\
\hline 1000 & 0.107 & 0.113 & 0.302 & 0.417 & 0.214 & 0.988 & 0.225 & 0.269 & \\
\hline 5000 & 0.050 & 0.048 & 0.128 & 0.179 & 0.090 & 0.353 & 0.097 & 0.102 & \\
\hline 25000 & 0.022 & 0.022 & 0.064 & 0.089 & 0.040 & 0.154 & 0.044 & 0.038 & \\
\hline 250 & 0.237 & 0.262 & 0.707 & 1.113 & 0.904 & 5.941 & 0.930 & 0.198 & $\rho=.9$ \\
\hline 500 & 0.157 & 0.162 & 0.491 & 0.726 & 0.362 & 1.889 & 0.382 & 0.106 & \\
\hline 1000 & 0.112 & 0.112 & 0.314 & 0.461 & 0.216 & 0.988 & 0.225 & 0.038 & \\
\hline 5000 & 0.048 & 0.048 & 0.130 & 0.193 & 0.091 & 0.360 & 0.096 & 0.010 & \\
\hline
\end{tabular}

Table 6: Root Mean Squared Error of CAM Parameter Estimates 


\begin{tabular}{|c|c|c|c|c|c|c|c|c|c|}
\hline \multirow{3}{*}{$\mathrm{N}$} & \multirow{3}{*}{$\begin{array}{c}U_{A, 1} \\
0 \\
\end{array}$} & \multicolumn{2}{|c|}{ A's Utilities } & \multirow{3}{*}{$\begin{array}{c}U_{A, 6} \\
-4.5\end{array}$} & \multicolumn{3}{|c|}{ B's Utilities } & \multirow{3}{*}{\multicolumn{2}{|c|}{$\rho$}} \\
\hline & & $U_{A, 3}$ & $U_{A, 5}$ & & $U_{B, 3}$ & $U_{B, 5}$ & $U_{B, 6}$ & & \\
\hline & & 6 & -4 & & 0 & 4 & -6 & & \\
\hline CAM & 0.002 & 6.016 & -3.994 & -4.498 & 0.003 & 4.012 & -6.051 & 0.057 & $\rho=.05$ \\
\hline SAM & -0.002 & 5.322 & -3.826 & -4.288 & 0.069 & 3.891 & -5.677 & & \\
\hline IAM & -0.002 & 6.022 & -4.024 & -4.525 & 0.003 & 4.027 & -6.037 & & \\
\hline CAM & 0.001 & 6.012 & -3.998 & -4.504 & 0.002 & 4.005 & -6.059 & 0.367 & $\rho=.35$ \\
\hline SAM & -0.023 & 5.385 & -3.972 & -4.502 & 0.058 & 3.912 & -6.151 & & \\
\hline IAM & -0.028 & 6.030 & -4.190 & -4.681 & -0.001 & 4.097 & -5.960 & & \\
\hline CAM & 0.004 & 6.025 & -4.008 & -4.518 & 0.000 & 4.004 & -6.067 & 0.657 & $\rho=.65$ \\
\hline SAM & -0.039 & 5.430 & -4.051 & -4.600 & 0.050 & 3.958 & -6.281 & & \\
\hline IAM & -0.042 & 6.014 & -4.265 & -4.752 & -0.016 & 4.112 & -5.942 & & \\
\hline CAM & -0.001 & 6.021 & -3.989 & -4.489 & -0.007 & 4.003 & -6.045 & 0.886 & $\rho=.95$ \\
\hline SAM & -0.041 & 5.408 & -4.004 & -4.526 & 0.030 & 3.958 & -6.187 & & \\
\hline IAM & -0.050 & 6.020 & -4.218 & -4.696 & -0.034 & 4.097 & -5.970 & & \\
\hline
\end{tabular}

Table 7: Mean Parameter Estimate Comparisons from CAM, SAM, and IAM 


\begin{tabular}{|c|c|c|c|c|c|c|c|c|c|}
\hline & $\mathrm{p}_{2}$ & RMSE & $\mathrm{p}_{4}$ & RMSE & $\mathrm{p}_{6}^{\mathrm{B}}$ & RMSE & $p_{6}$ & RMSE & $\rho$ \\
\hline DGP & 0.375 & & 0.627 & & 0.360 & & 0.362 & & $\rho=.05$ \\
\hline CAM & 0.375 & 0.035 & 0.628 & 0.012 & 0.358 & 0.017 & 0.361 & 0.016 & \\
\hline SAM & 0.352 & 0.092 & 0.659 & 0.052 & 0.348 & 0.049 & 0.373 & 0.040 & \\
\hline IAM & 0.382 & 0.034 & 0.623 & 0.011 & & & 0.361 & 0.017 & \\
\hline DGP & 0.206 & & 0.697 & & 0.336 & & 0.362 & & $\rho=.35$ \\
\hline CAM & 0.195 & 0.059 & 0.706 & 0.035 & 0.330 & 0.021 & 0.360 & 0.012 & \\
\hline SAM & 0.184 & 0.093 & 0.729 & 0.067 & 0.309 & 0.062 & 0.355 & 0.038 & \\
\hline IAM & 0.310 & 0.108 & 0.638 & 0.060 & & & 0.364 & 0.018 & \\
\hline DGP & 0.023 & & 0.865 & & 0.260 & & 0.362 & & $\rho=.65$ \\
\hline CAM & 0.031 & 0.026 & 0.858 & 0.039 & 0.261 & 0.028 & 0.359 & 0.010 & \\
\hline SAM & 0.094 & 0.100 & 0.786 & 0.100 & 0.285 & 0.065 & 0.350 & 0.041 & \\
\hline IAM & 0.279 & 0.257 & 0.644 & 0.221 & & & 0.366 & 0.018 & \\
\hline DGP & 0.129 & & 0.762 & & 0.311 & & 0.362 & & $\rho=.95$ \\
\hline CAM & 0.117 & 0.056 & 0.776 & 0.042 & 0.303 & 0.027 & 0.362 & 0.011 & \\
\hline SAM & 0.162 & 0.089 & 0.739 & 0.060 & 0.309 & 0.050 & 0.357 & 0.035 & \\
\hline IAM & 0.316 & 0.189 & 0.636 & 0.126 & & & 0.368 & 0.018 & \\
\hline
\end{tabular}

Table 8: Mean Probability Estimate and RMSE Comparisons from CAM, SAM, and IAM 


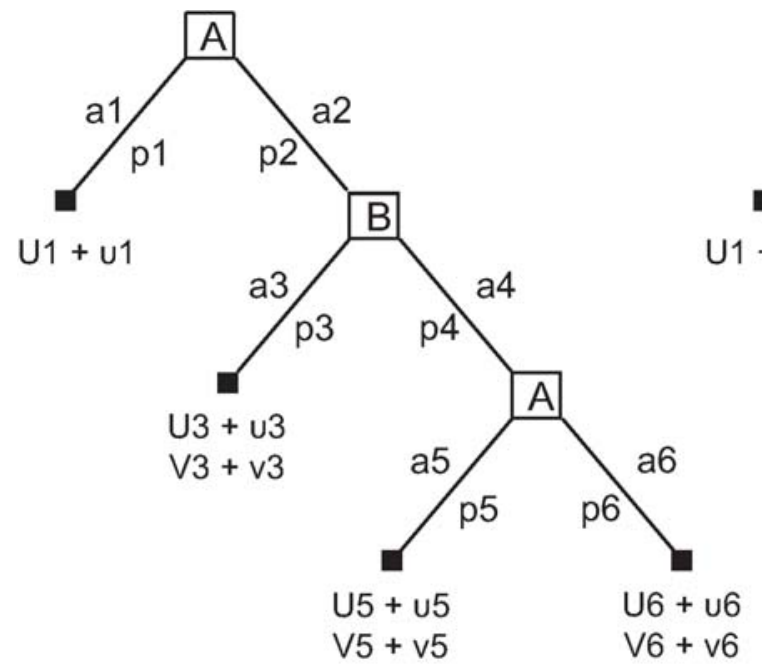

(a) Single Agent Model

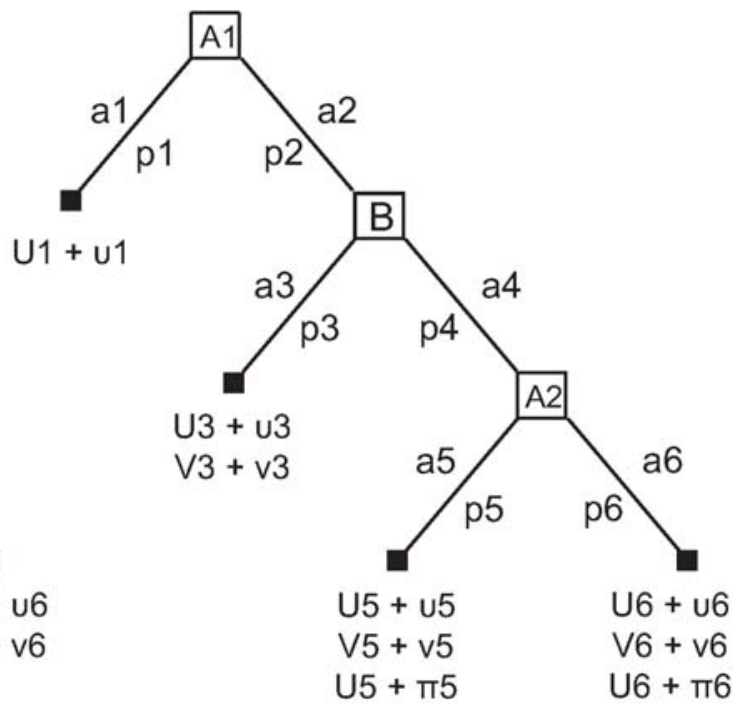

(b) Correlated Agent Model

Figure 1: Two-Player Signalling Game 


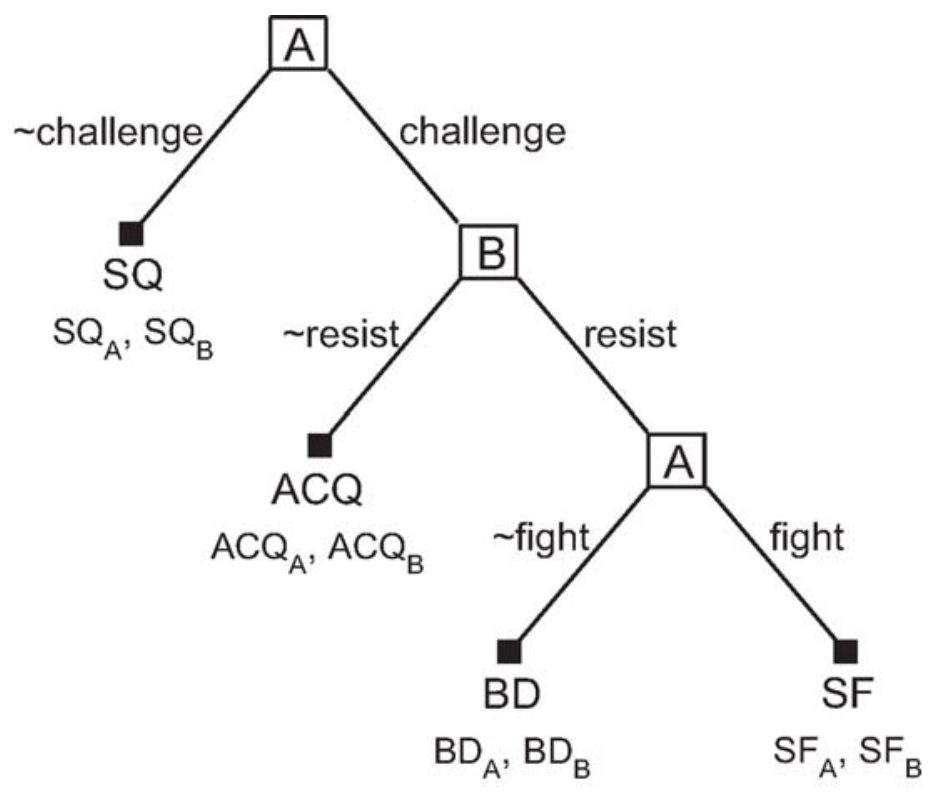

Figure 2: Lewis and Schultz Model of Crisis Bargaining 


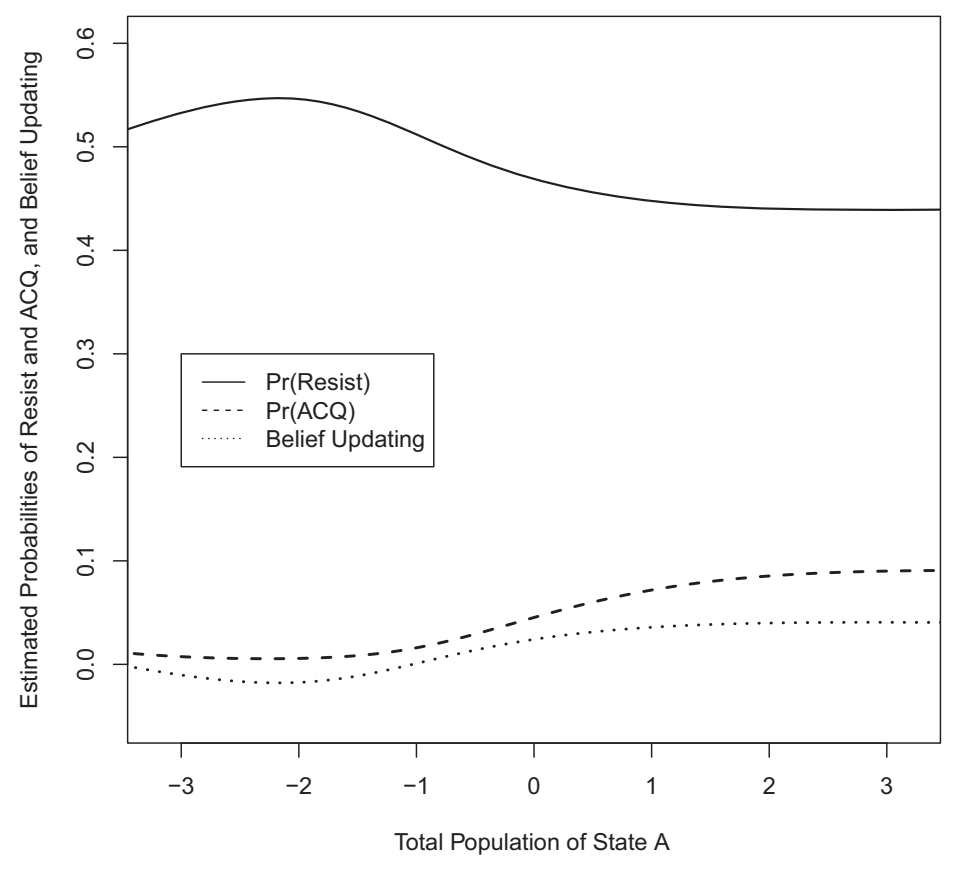

Figure 3: Estimated Probabilities of Resist, ACQ, and Belief Updating 


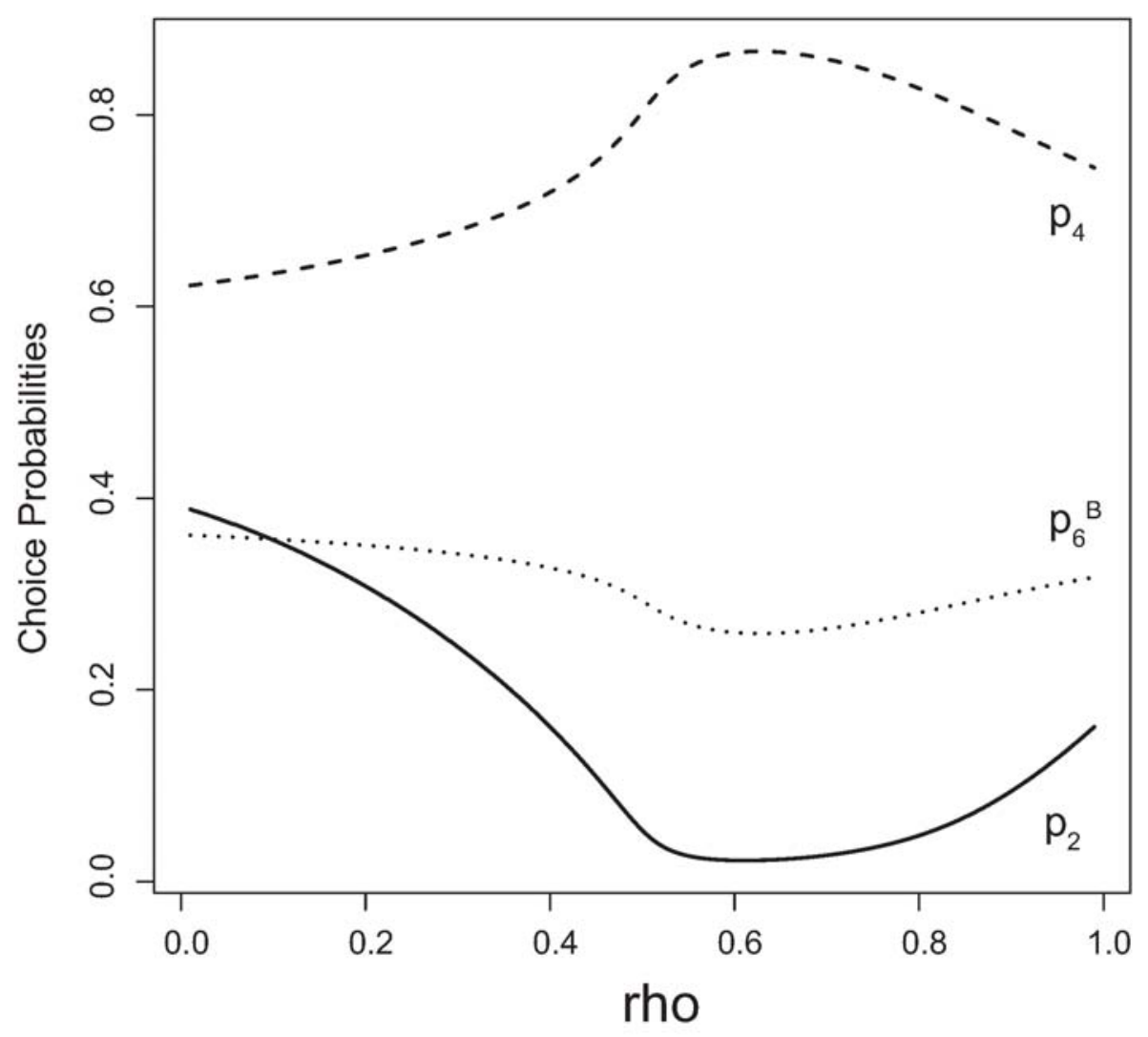

Figure 4: Action Probabilities as a Function of $\rho$ 